\title{
BLOCKS OF CYCLOTOMIC HECKE ALGEBRAS AND KHOVANOV-LAUDA ALGEBRAS
}

\author{
JONATHAN BRUNDAN AND ALEXANDER KLESHCHEV
}

\begin{abstract}
We construct an explicit isomorphism between blocks of cyclotomic Hecke algebras and (sign-modified) cyclotomic Khovanov-Lauda algebras in type A. These isomorphisms connect the categorification conjecture of Khovanov and Lauda to Ariki's categorification theorem. The Khovanov-Lauda algebras are naturally graded, which allows us to exhibit a non-trivial $\mathbb{Z}$-grading on blocks of cyclotomic Hecke algebras, including symmetric groups in positive characteristic.
\end{abstract}

\section{INTRODUCTION}

In KL1, KL2, Khovanov and Lauda have introduced a remarkable new family of algebras and formulated a categorification conjecture predicting a tight connection between the representation theory of these algebras and Lusztig's geometric construction of canonical bases [L2]. This paper arose as a first attempt to understand the cyclotomic Khovanov-Lauda algebras in type A by relating them to cyclotomic Hecke algebras and their rational degenerations.

Let $F$ be a fixed ground field and $q \in F^{\times}$. Let $e$ be the smallest positive integer such that $1+q+\cdots+q^{e-1}=0$, setting $e:=0$ if no such integer exists. The main result of the article gives an explicit isomorphism between blocks of cyclotomic Hecke algebras associated to the complex reflection groups of type $G(l, 1, d)$ if $q \neq 1$, or the corresponding degenerate cyclotomic Hecke algebras if $q=1$, and a sign-modified version of cyclotomic Khovanov-Lauda algebras of type $\mathrm{A}_{\infty}$ if $e=0$ or type $\mathrm{A}_{e-1}^{(1)}$ if $e>0$.

For $F$ of characteristic zero, this isomorphism connects the categorification conjecture of Khovanov and Lauda [KL1, §3.4] in type A to Ariki's categorification theorem $[\mathrm{A}$ and its degenerate analogue [BK]. It doesn't immediately prove the Khovanov-Lauda conjecture in any of these cases, because the conjecture takes into account a natural $\mathbb{Z}$-grading on cyclotomic Khovanov-Lauda algebras which is hard to identify on Hecke algebras.

To formulate the main results precisely, let $\Gamma$ be the quiver with vertex set $I:=\mathbb{Z} / e \mathbb{Z}$, and a directed edge from $i$ to $j$ if $j=i+1$. Thus $\Gamma$ is the quiver

2000 Mathematics Subject Classification: $20 \mathrm{C} 08$.

Supported in part by NSF grant DMS-0654147. This work was started at MSRI. 
of type $\mathrm{A}_{\infty}$ if $e=0$ or $\mathrm{A}_{e-1}^{(1)}$ if $e>0$, with a specific orientation:

$$
\begin{aligned}
& \mathrm{A}_{\infty}: \quad \cdots \longrightarrow-2 \longrightarrow-1 \longrightarrow 0 \longrightarrow 1 \longrightarrow 2 \longrightarrow \cdots
\end{aligned}
$$

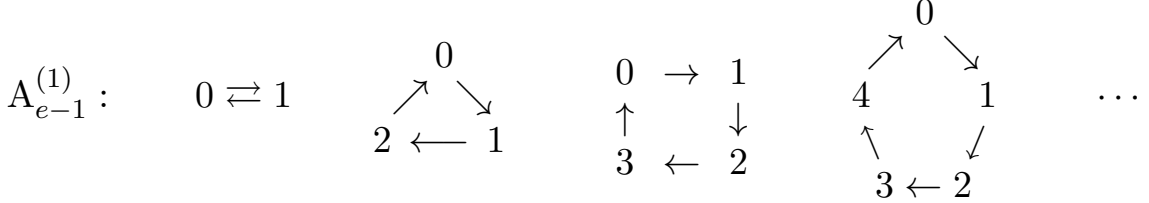

The corresponding (symmetric) Cartan matrix $\left(a_{i, j}\right)_{i, j \in I}$ is defined by

$$
a_{i, j}:=\left\{\begin{aligned}
2 & \text { if } i=j, \\
0 & \text { if } i+j, \\
-1 & \text { if } i \rightarrow j \text { or } i \leftarrow j, \\
-2 & \text { if } i \rightleftarrows j .
\end{aligned}\right.
$$

Here the symbols $i \rightarrow j$ and $j \leftarrow i$ both indicate that $j=i+1 \neq i-1, i \rightleftarrows j$ indicates that $j=i+1=i-1$, and $i+j$ indicates that $j \neq i, i \pm 1$.

To the index set $I$, we associate two lattices

$$
P:=\bigoplus_{i \in I} \mathbb{Z} \Lambda_{i}, \quad Q:=\bigoplus_{i \in I} \mathbb{Z} \alpha_{i},
$$

and let $(.,):. P \times Q \rightarrow \mathbb{Z}$ be the bilinear pairing defined by $\left(\Lambda_{i}, \alpha_{j}\right):=\delta_{i, j}$. Let $P_{+}$(resp. $Q_{+}$) denote the subset of $P$ (resp. $Q$ ) consisting of the elements that have non-negative coefficients when written in terms of the given basis. For $\alpha \in Q_{+}$and $\Lambda \in P_{+}$define the height of $\alpha$ and the level of $\Lambda$ as follows:

$$
\operatorname{ht}(\alpha):=\sum_{i \in I}\left(\Lambda_{i}, \alpha\right), \quad l(\Lambda):=\sum_{i \in I}\left(\Lambda, \alpha_{i}\right) .
$$

Let $S_{d}$ be the symmetric group with basic transpositions $s_{1}, \ldots, s_{d-1}$. It acts on the left on the set of $d$-tuples $\boldsymbol{i}=\left(i_{1}, \ldots, i_{d}\right) \in I^{d}$ by place permutation.

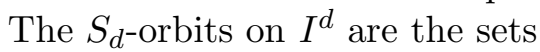

$$
I^{\alpha}:=\left\{\boldsymbol{i}=\left(i_{1}, \ldots, i_{d}\right) \in I^{d} \mid \alpha_{i_{1}}+\cdots+\alpha_{i_{d}}=\alpha\right\}
$$

parametrized by all $\alpha \in Q_{+}$of height $d$.

Let $H_{d}$ denote the affine Hecke algebra associated to $S_{d}$ if $q \neq 1$, or its rational degeneration if $q=1$. Thus, $H_{d}$ is the $F$-algebra defined by generators $T_{1}, \ldots, T_{d-1}, X_{1}^{ \pm 1}, \ldots, X_{d}^{ \pm 1}$ and relations (4.2)-(4.5) if $q \neq 1$, or by generators $s_{1}, \ldots, s_{d-1}, x_{1}, \ldots, x_{d}$ and relations (3.2)-(3.5) if $q=1$. From now on, fix $\Lambda \in P_{+}$of level $l$ and let $H_{d}^{\Lambda}$ be the corresponding cyclotomic quotient of $H_{d}$. Thus,

$$
H_{d}^{\Lambda}:= \begin{cases}H_{d} /\left\langle\prod_{i \in I}\left(X_{1}-q^{i}\right)^{\left(\Lambda, \alpha_{i}\right)}\right\rangle & \text { if } q \neq 1, \\ H_{d} /\left\langle\prod_{i \in I}\left(x_{1}-i\right)^{\left(\Lambda, \alpha_{i}\right)}\right\rangle & \text { if } q=1 .\end{cases}
$$

We refer to this algebra simply as the cyclotomic Hecke algebra if $q \neq 1$ and the degenerate cyclotomic Hecke algebra if $q=1$.

There is a natural system $\left\{e(\boldsymbol{i}) \mid \boldsymbol{i} \in I^{d}\right\}$ of mutually orthogonal idempotents in $H_{d}^{\Lambda}$; see 4.1 or 3.1 for more details in the two cases. In addition to 
$\Lambda \in P_{+}$, fix also $\alpha \in Q_{+}$of height $d$. Let

$$
e_{\alpha}:=\sum_{i \in I^{\alpha}} e(i) \in H_{d}^{\Lambda} .
$$

As a consequence of $[\mathrm{LM}]$ or $\left[\mathrm{B}\right.$, Theorem 1], $e_{\alpha}$ is either zero or it is a primitive central idempotent in $H_{d}^{\Lambda}$. Hence the algebra

$$
H_{\alpha}^{\Lambda}:=e_{\alpha} H_{d}^{\Lambda}
$$

is either zero or it is a single block of the algebra $H_{d}^{\Lambda}$. For $h \in H_{d}^{\Lambda}$, we still write $h$ for the projection $e_{\alpha} h \in H_{\alpha}^{\Lambda}$. So $H_{\alpha}^{\Lambda}$ again has generators $T_{1}, \ldots, T_{d-1}, X_{1}^{ \pm 1}, \ldots, X_{d}^{ \pm 1}$ if $q \neq 1$, or $s_{1}, \ldots, s_{d-1}, x_{1}, \ldots, x_{d}$ if $q=1$. However, it seems difficult to describe a complete set of relations between these generators at the level of $H_{\alpha}^{\Lambda}$. These generators are also not well-suited for defining interesting gradings. So, inspired by [KL1, KL2], we introduce an explicit new set of generators

$$
\left\{e(\boldsymbol{i}) \mid \boldsymbol{i} \in I^{\alpha}\right\} \cup\left\{y_{1}, \ldots, y_{d}\right\} \cup\left\{\psi_{1}, \ldots, \psi_{d-1}\right\}
$$

of $H_{\alpha}^{\Lambda}$, which we call the Khovanov-Lauda generators; see (4.21) and (4.38) or (3.21) and (3.32) in the two cases.

Main Theorem. The algebra $H_{\alpha}^{\Lambda}$ is generated by the elements (1.5) subject only to the following relations for $\boldsymbol{i}, \boldsymbol{j} \in I^{\alpha}$ and all admissible $r, s$ :

$$
\begin{aligned}
& y_{1}^{\left(\Lambda, \alpha_{i_{1}}\right)} e(\boldsymbol{i})=0 \\
& e(\boldsymbol{i}) e(\boldsymbol{j})=\delta_{\boldsymbol{i}, \boldsymbol{j}} e(\boldsymbol{i}) ; \quad \sum_{\boldsymbol{i} \in I^{\alpha}} e(\boldsymbol{i})=1 ; \\
& y_{r} e(\boldsymbol{i})=e(\boldsymbol{i}) y_{r} ; \quad \psi_{r} e(\boldsymbol{i})=e\left(s_{r} \cdot \boldsymbol{i}\right) \psi_{r} ; \\
& y_{r} y_{s}=y_{s} y_{r} ; \\
& \psi_{r} y_{s}=y_{s} \psi_{r} \quad \text { if } s \neq r, r+1 \text {; } \\
& \psi_{r} \psi_{s}=\psi_{s} \psi_{r} \\
& \psi_{r} y_{r+1} e(\boldsymbol{i})= \begin{cases}\left(y_{r} \psi_{r}+1\right) e(\boldsymbol{i}) & \text { if } i_{r}=i_{r+1}, \\
y_{r} \psi_{r} e(\boldsymbol{i}) & \text { if } i_{r} \neq i_{r+1}\end{cases} \\
& y_{r+1} \psi_{r} e(\boldsymbol{i})=\left\{\begin{array}{ll}
\left(\psi_{r} y_{r}+1\right) e(\boldsymbol{i}) & \text { if } i_{r}=i_{r+1}, \\
\psi_{r} y_{r} e(\boldsymbol{i}) & \text { if } i_{r} \neq i_{r+1}
\end{array} ;\right. \\
& \psi_{r}^{2} e(\boldsymbol{i})= \begin{cases}0 & \text { if } i_{r}=i_{r+1}, \\
e(\boldsymbol{i}) & \text { if } i_{r}+i_{r+1}, \\
\left(y_{r+1}-y_{r}\right) e(\boldsymbol{i}) & \text { if } i_{r} \rightarrow i_{r+1}, \\
\left(y_{r}-y_{r+1}\right) e(\boldsymbol{i}) & \text { if } i_{r} \leftarrow i_{r+1}, \\
\left(y_{r+1}-y_{r}\right)\left(y_{r}-y_{r+1}\right) e(\boldsymbol{i}) & \text { if } i_{r} \rightleftarrows i_{r+1}\end{cases} \\
& \psi_{r} \psi_{r+1} \psi_{r} e(\boldsymbol{i})=\left\{\begin{array}{cl}
\left(\psi_{r+1} \psi_{r} \psi_{r+1}+1\right) e(\boldsymbol{i}) & \text { if } i_{r+2}=i_{r} \rightarrow i_{r+1}, \\
\left(\psi_{r+1} \psi_{r} \psi_{r+1}-1\right) e(\boldsymbol{i}) & \text { if } i_{r+2}=i_{r} \leftarrow i_{r+1}, \\
\left(\psi_{r+1} \psi_{r} \psi_{r+1}-2 y_{r+1}\right. & \\
\left.+y_{r}+y_{r+2}\right) e(\boldsymbol{i}) & \text { if } i_{r+2}=i_{r} \rightleftarrows i_{r+1}, \\
\psi_{r+1} \psi_{r} \psi_{r+1} e(\boldsymbol{i}) & \text { otherwise. }
\end{array}\right.
\end{aligned}
$$

A striking feature of the above theorem is that the relations depend only on the quiver $\Gamma$ (hence $e$ ) but do not involve the parameter $q$. 
Corollary 1. There is a unique $\mathbb{Z}$-grading on $H_{\alpha}^{\Lambda}$ such that e $(\boldsymbol{i})$ is of degree $0, y_{r}$ is of degree 2 , and $\psi_{r} e(\boldsymbol{i})$ is of degree $-a_{i_{r}, i_{r+1}}$ for each $r$ and $\boldsymbol{i} \in I^{\alpha}$.

Corollary 2. Suppose that $F$ is of characteristic zero. Then the algebra $H_{d}^{\Lambda}$ for $q$ not a root of unity is isomorphic to the algebra $H_{d}^{\Lambda}$ for $q=1$. In other words, the cyclotomic Hecke algebra for generic $q$ is isomorphic to its rational degeneration.

The presentation for the algebra $H_{\alpha}^{\Lambda}$ in our Main Theorem is a signmodified version of the presentation for the cyclotomic Khovanov-Lauda algebra associated to the quiver $\Gamma$ and the weight $\Lambda$ as defined in [KL1, §3.4]; this sign-modified version was introduced already in KL2 (except in the case $e=2$ ). Thus the theorem shows that blocks of cyclotomic Hecke algebras are cyclotomic Khovanov-Lauda algebras.

If $\Lambda$ is of level one, $H_{d}^{\Lambda}$ is isomorphic to the group algebra $F S_{d}$ of the symmetric group if $q=1$ or the associated Iwahori-Hecke algebra for arbitrary $q$. In these cases, our Main Theorem for $e=0$ is essentially Young's semi-normal form (see $\S 5$ ), while for $e>0$ Corollary 1 yields interesting $\mathbb{Z}$-gradings on blocks of symmetric groups and the associated Iwahori-Hecke algebras. The existence of such gradings was predicted already by Rouquier R1, Remark 3.11] and Turner [T]. This means that it is now possible to study graded representation theory of these algebras. First steps in this direction are taken in [BKW], in which we construct graded versions of Specht modules.

Corollary 2 can be viewed as an extension of Lusztig's results from [L1] in type A. As an application, it is easy to see that Ariki's categorification theorem from $\mathrm{A}$ when $q$ is not a root of unity is simply equivalent to the degenerate analogue proved (in a quite different way) in [BK]. Our Main Theorem also makes possible the comparison of blocks of cyclotomic Hecke algebras at a primitive complex $p$ th root of unity with corresponding blocks of degenerate cyclotomic Hecke algebras over fields of characteristic $p$; see $\S 6$ for further discussion.

Before we started work on this article (and before [KL1] became available), the first author jointly with Stroppel made calculations as part of [BS] that are equivalent to the Main Theorem for $\Lambda$ of level two, $q=1$ and $e=0$. In that case, the algebras $H_{\alpha}^{\Lambda}$ are Morita equivalent to (slightly generalised versions of) Khovanov's diagram algebra. This connection is exploited further in [BS] to give an elementary proof of the Khovanov-Lauda categorification conjecture for level two weights in type $\mathrm{A}_{\infty}$.

Since completing this work, we have learnt from Rouquier that he has independently discovered essentially the same family of algebras as Khovanov and Lauda at the affine level; he refers to them as quiver nil-Hecke algebras. In particular, in [R2, §3.2.6], Rouquier has proved an analogue of our Main Theorem for the affine algebras (suitably localized).

The rest of the article is taken up with the proof of the Main Theorem. The strategy is clear: let $R_{\alpha}^{\Lambda}$ be the algebra defined by generators and relations as in the Main Theorem. Then we need to construct mutually inverse homomorphisms $\rho: R_{\alpha}^{\Lambda} \rightarrow H_{\alpha}^{\Lambda}$ and $\sigma: H_{\alpha}^{\Lambda} \rightarrow R_{\alpha}^{\Lambda}$ by explicitly checking relations in both directions. However, there are many subtle differences in the 
two cases $q \neq 1$ and $q=1$, so we carry out the two sets of calculations independently, treating the easier degenerate case in $\S 3$ then the non-degenerate case in $\S 4$. In $\S 2$ we make a few definitions that are common to both cases. Finally in $\S 5$ and $\S 6$ we discuss briefly the relation to Young's semi-normal form and make some observations about base change.

Acknowledgements. We thank Anton Cox for pointing out a gap in an earlier version, and Meinolf Geck for drawing our attention to the fact that our results could be used to prove a conjecture of Mathas.

\section{Preliminaries}

2.1. Divided difference operators. The symmetric group $S_{d}$ acts on the left on the polynomial ring $F\left[y_{1}, \ldots, y_{d}\right]$ and on the ring of power series $F\left[\left[y_{1}, \ldots, y_{d}\right]\right]$ by permuting variables; we often denote $w \cdot f$ by ${ }^{w} f$ for $w \in S_{d}$ and $f \in F\left[\left[y_{1}, \ldots, y_{d}\right]\right]$. For any $f \in F\left[y_{1}, \ldots, y_{d}\right]$ and $1 \leq r<d$, the divided difference operator $\partial_{r}$ is defined by

$$
\partial_{r}(f):=\frac{{ }^{s_{r}} f-f}{y_{r}-y_{r+1}}
$$

It extends to $F\left[\left[y_{1}, \ldots, y_{d}\right]\right]$ by continuity. We will need the product rule for divided difference operators: for $f, g \in F\left[\left[y_{1}, \ldots, y_{d}\right]\right]$ we have that

$$
\partial_{r}(f g)=\partial_{r}(f) g+{ }^{s_{r}} f \partial_{r}(g)=\partial_{r}(f)^{s_{r}} g+f \partial_{r}(g) .
$$

Note as a matter of notation here that ${ }^{s_{r}} f g$ means $\left({ }^{s_{r}} f\right) g$ not ${ }^{s_{r}}(f g)$.

We will often be given an $F$-algebra $A$ and commuting nilpotent elements $y_{1}, \ldots, y_{d} \in A$. There is then an algebra homomorphism

$$
F\left[\left[y_{1}, \ldots, y_{d}\right]\right] \rightarrow A
$$

mapping each $y_{r}$ to the element of $A$ with the same name. Given also power series $f, g \in F\left[\left[y_{1}, \ldots, y_{d}\right]\right]$, this homomorphism allows us to interpret expressions like ${ }^{s_{r}} f, \partial_{r}(g)$ and $f / g$ as elements of $A$; we mean the element of $A$ obtained by first evaluating the given expression as a power series in $F\left[\left[y_{1}, \ldots, y_{d}\right]\right]$ and only then taking the image of the result under the homomorphism (2.3). We stress that $f$ and $g$ must be given specifically as power series (not merely as elements of $A$ ) for such expressions to make sense.

2.2. Cyclotomic Khovanov-Lauda algebras. For the remainder of the article, we fix notation exactly as in the statement of the Main Theorem. In particular, $\Gamma$ is the quiver with vertex set $I=\mathbb{Z} / e \mathbb{Z}$ defined at the beginning of the introduction and $P, Q$ are as in (1.1). The (sign-modified) cyclotomic Khovanov-Lauda algebra of type $\Gamma$ associated to $\Lambda \in P_{+}$of level $l$ and $\alpha \in Q_{+}$ of height $d$ is the $F$-algebra $R_{\alpha}^{\Lambda}$ defined by the generators

$$
\left\{e(\boldsymbol{i}) \mid \boldsymbol{i} \in I^{\alpha}\right\} \cup\left\{y_{1}, \ldots, y_{d}\right\} \cup\left\{\psi_{1}, \ldots, \psi_{d-1}\right\}
$$

subject to the relations (1.6)-(1.15) above.

In view of the first lemma below, we are in the situation of (2.3), so have a homomorphism

$$
F\left[\left[y_{1}, \ldots, y_{d}\right]\right] \rightarrow R_{\alpha}^{\Lambda}
$$


mapping $y_{1}, \ldots, y_{d}$ to the elements of $R_{\alpha}^{\Lambda}$ with the same name. By (1.10), (1.12) and (1.13), the following useful identity holds in $R_{\alpha}^{\Lambda}$ :

$$
f \psi_{r} e(\boldsymbol{i})= \begin{cases}\psi_{r}^{s_{r}} f e(\boldsymbol{i})+\partial_{r}(f) e(\boldsymbol{i}) & \text { if } i_{r}=i_{r+1}, \\ \psi_{r}{ }^{s_{r}} f e(\boldsymbol{i}) & \text { otherwise }\end{cases}
$$

for any $1 \leq r<d, \boldsymbol{i} \in I^{\alpha}$ and $f \in F\left[\left[y_{1}, \ldots, y_{d}\right]\right]$.

Lemma 2.1. The elements $y_{r} \in R_{\alpha}^{\Lambda}$ are nilpotent for all $1 \leq r \leq d$.

Proof. It suffices to prove that each $y_{r} e(\boldsymbol{i})$ is nilpotent. Apply induction on $r$. The base case $r=1$ follows as $\left(y_{1} e(\boldsymbol{i})\right)^{\left(\Lambda, \alpha_{i_{1}}\right)}=0$ in $R_{\alpha}^{\Lambda}$. For the induction step, we assume that $y_{r}$ is nilpotent for some $1 \leq r<d$ and prove that $y_{r+1} e(\boldsymbol{i})$ is too. We consider two cases.

If $i_{r}=i_{r+1}$, set $\tau_{r}:=\psi_{r}\left(y_{r}-y_{r+1}\right)+1$. One checks that $\tau_{r}^{2} e(\boldsymbol{i})=e(\boldsymbol{i})$ and $\tau_{r} y_{r} \tau_{r} e(\boldsymbol{i})=y_{r+1} e(\boldsymbol{i})$. Hence $y_{r+1}^{n} e(\boldsymbol{i})=\tau_{r} y_{r}^{n} \tau_{r} e(\boldsymbol{i})$, and the nilpotency of $y_{r+1} e(\boldsymbol{i})$ follows from that of $y_{r}$.

If $i_{r} \neq i_{r+1}$, then multiplying the equation $y_{r} \psi_{r} e(\boldsymbol{i})=\psi_{r} y_{r+1} e(\boldsymbol{i})$ on the left by $\psi_{r}$ and using (1.14) gives that $\psi_{r} y_{r} \psi_{r} e(\boldsymbol{i})= \pm\left(y_{r}-y_{r+1}\right)^{k} y_{r+1} e(\boldsymbol{i})$ for some $k \in\{0,1,2\}$ and some choice of sign. Hence

$$
y_{r+1}^{k+1} e(\boldsymbol{i})=y_{r} f e(\boldsymbol{i}) \pm \psi_{r} y_{r} \psi_{r} e(\boldsymbol{i})
$$

for some (possibly zero) $f \in F\left[y_{r}, y_{r+1}\right]$ and some sign. As $y_{r}$ is nilpotent, we deduce using (1.14) that $\psi_{r} y_{r} \psi_{r} e(\boldsymbol{i})$ is nilpotent too, and of course $y_{r} f$ is nilpotent as well. Observing finally that $y_{r} f e(\boldsymbol{i})$ and $\psi_{r} y_{r} \psi_{r} e(\boldsymbol{i})$ commute, this implies the nilpotency of $y_{r+1}^{k+1} e(\boldsymbol{i})$. Hence $y_{r+1} e(\boldsymbol{i})$ is nilpotent too.

Corollary 2.2. $R_{\alpha}^{\Lambda}$ is a finite dimensional algebra.

Proof. For each $w \in S_{d}$, fix a reduced expression $w=s_{i_{1}} \cdots s_{i_{n}}$ and then define $\psi_{w}:=\psi_{i_{1}} \cdots \psi_{i_{n}}$. By an easy application of the relations (1.7)-(1.15) one checks as in [KL1, §2.3] that $R_{\alpha}^{\Lambda}$ is spanned by the elements

$$
\left\{\psi_{w} y_{1}^{n_{1}} \cdots y_{d}^{n_{d}} e(\boldsymbol{i}) \mid w \in S_{d}, \boldsymbol{i} \in I^{\alpha}, n_{1}, \ldots, n_{d} \geq 0\right\} .
$$

It remains to observe by Lemma 2.1 that all but finitely many of these elements are zero.

The following conjecture is a quite trivial consequence of the relations in the case $l=1$, and is established for $l=2$ in [BS]. We couldn't see how to check even the $l=2$ case using the relations alone.

Conjecture 2.3. If $e=0$ and $\Lambda$ is of level $l$ then $y_{r}^{l}=0$ in $R_{\alpha}^{\Lambda}$ for any $1 \leq r \leq d$.

\section{The Degenerate CASE}

3.1. Blocks of degenerate cyclotomic Hecke algebras. For $d \geq 0$, let $H_{d}$ be the degenerate affine Hecke algebra, working always over the fixed field $F$ of characteristic $e \geq 0$. So $H_{d}$ has generators

$$
\left\{x_{1}, \ldots, x_{d}\right\} \cup\left\{s_{1}, \ldots, s_{d-1}\right\}
$$


subject to the following relations for all admissible indices:

$$
\begin{array}{rlrl}
x_{r} x_{s} & =x_{s} x_{r} ; & & \\
s_{r} x_{r+1} & =x_{r} s_{r}+1, & & s_{r} x_{s}=x_{s} s_{r} \quad \text { if } s \neq r, r+1 ; \\
s_{r}^{2} & =1 ; & & \\
s_{r} s_{r+1} s_{r} & =s_{r+1} s_{r} s_{r+1}, & s_{r} s_{t}=s_{t} s_{r} \quad \text { if }|r-t|>1 .
\end{array}
$$

Given $\Lambda \in P_{+}$of level $l$, let $H_{d}^{\Lambda}$ be the cyclotomic quotient from (1.2). The elements $x_{1}^{m_{1}} \cdots x_{d}^{m_{d}} w$ for $0 \leq m_{1}, \ldots, m_{d}<l$ and $w \in S_{d}$ give a basis for $H_{d}^{\Lambda}$; see e.g. [K1, Theorem 7.5.6]. Hence:

$$
\operatorname{dim} H_{d}^{\Lambda}=l^{d} d !
$$

For example, if $l=1$ then $H_{d}^{\Lambda} \cong F S_{d}$.

Let $M$ be a finite dimensional $H_{d}^{\Lambda}$-module. By [K1, Lemma 7.1.2], the eigenvalues of each $x_{r}$ on $M$ belong to $I \subseteq F$. So $M$ decomposes as the direct sum $M=\bigoplus_{\boldsymbol{i} \in I^{d}} M_{\boldsymbol{i}}$ of its weight spaces

$$
M_{i}:=\left\{v \in M \mid\left(x_{r}-i_{r}\right)^{N} v=0 \text { for all } r=1, \ldots, d \text { and } N \gg 0\right\} .
$$

Note also by [K1, Lemma 2.2.1] that

$$
s_{r}\left(M_{i}\right) \subseteq M_{\boldsymbol{i}}+M_{s_{r} \cdot \boldsymbol{i}}
$$

for each $1 \leq r<d$. Considering the weight space decomposition of the regular module, we deduce that there is a system $\left\{e(\boldsymbol{i}) \mid \boldsymbol{i} \in I^{d}\right\}$ of mutually orthogonal idempotents in $H_{d}^{\Lambda}$ such that $e(\boldsymbol{i}) M=M_{\boldsymbol{i}}$ for each finite dimensional module $M$. In fact, each $e(\boldsymbol{i})$ lies in the commutative subalgebra generated by $x_{1}, \ldots, x_{d}$. All but finitely many of the $e(\boldsymbol{i})$ 's are zero, and their sum is the identity element in $H_{d}^{\Lambda}$.

By [B, Theorem 1], the center $Z\left(H_{d}^{\Lambda}\right)$ consists of all symmetric polynomials in $x_{1}, \ldots, x_{d}$. So, given also $\alpha \in Q_{+}$of height $d$, the idempotent $e_{\alpha}$ from (1.3) is either zero or it is a primitive central idempotent in $H_{d}^{\Lambda}$. This means that the algebra $H_{\alpha}^{\Lambda}:=e_{\alpha} H_{d}^{\Lambda}$ from (1.4) is either zero or it is a block of $H_{d}^{\Lambda}$. The subalgebra of $H_{\alpha}^{\Lambda}$ generated by (the images of) $x_{1}, \ldots, x_{d}$ will be denoted $\operatorname{Pol}_{\alpha}^{\Lambda}$. Note $\operatorname{Pol}_{\alpha}^{\Lambda} e(\boldsymbol{i})$ is an algebra with identity element $e(\boldsymbol{i})$. If $x \in \operatorname{Pol}_{\alpha}^{\Lambda}$ is such that $x e(\boldsymbol{i})$ is a unit in $\operatorname{Pol}_{\alpha}^{\Lambda} e(\boldsymbol{i})$, we write $x^{-1} e(\boldsymbol{i})$ for its inverse in $\operatorname{Pol}_{\alpha}^{\Lambda} e(\boldsymbol{i})$ (interpreted as 0 if $e(\boldsymbol{i})=0$ ). For example, set

$$
x_{r, s}:=x_{r}-x_{s},
$$

and let $\boldsymbol{i} \in I^{\alpha}$ be such that $i_{r} \neq i_{s}$. Then $x_{r, s}^{-1} e(\boldsymbol{i})$ makes sense.

3.2. Intertwining elements $\varphi_{\boldsymbol{r}}$. We now introduce certain remarkable elements of $H_{\alpha}^{\Lambda}$ called intertwining elements: let

$$
\varphi_{r}:=s_{r}+\sum_{\substack{\boldsymbol{i} \in I^{\alpha} \\ i_{r} \neq i_{r+1}}} x_{r, r+1}^{-1} e(\boldsymbol{i})+\sum_{\substack{\boldsymbol{i} \in I^{\alpha} \\ i_{r}=i_{r+1}}} e(\boldsymbol{i})
$$

for $1 \leq r<d$. This is a slightly modified version of the usual intertwining element as in [Ro, §2] or [K1, (3.19)]:

$$
\theta_{r}:=s_{r} x_{r, r+1}+1 .
$$


The elements $\theta_{r}$ have the following nice properties [K1, §3.8] (cf. [L1, Proposition 5.2]):

$$
\begin{aligned}
\theta_{r}^{2} & =1-x_{r, r+1}^{2} ; & & \\
\theta_{r} x_{r+1} & =x_{r} \theta_{r}, \quad x_{r+1} \theta_{r}=\theta_{r} x_{r}, & & \theta_{r} x_{s}=x_{s} \theta_{r} \text { if } s \neq r, r+1 ; \\
\theta_{r} \theta_{r+1} \theta_{r} & =\theta_{r+1} \theta_{r} \theta_{r+1}, & & \theta_{r} \theta_{s}=\theta_{s} \theta_{r} \text { if }|r-s|>1 .
\end{aligned}
$$

The elements $\varphi_{r}$ inherit similar properties:

Lemma 3.1. The intertwining elements satisfy the following relations for all $\boldsymbol{i} \in I^{\alpha}$ and admissible $r, s:$

$$
\begin{aligned}
& \varphi_{r} e(\boldsymbol{i})=e\left(s_{r} \cdot \boldsymbol{i}\right) \varphi_{r} \\
& \varphi_{r} x_{s}=x_{s} \varphi_{r} \\
& \text { if } s \neq r, r+1 \text {; } \\
& \varphi_{r} \varphi_{s}=\varphi_{s} \varphi_{r} \\
& \text { if }|r-s|>1 \text {; } \\
& \varphi_{r} x_{r+1} e(\boldsymbol{i})=\left\{\begin{array}{l}
x_{r} \varphi_{r} e(\boldsymbol{i}) \\
\left(x_{r} \varphi_{r}+1-x_{r, r+1}\right) e(\boldsymbol{i})
\end{array}\right. \\
& \text { if } i_{r} \neq i_{r+1} \\
& x_{r+1} \varphi_{r} e(\boldsymbol{i})= \begin{cases}\varphi_{r} x_{r} e(\boldsymbol{i}) & \text { if } i_{r} \neq i_{r+1}, \\
\left(\varphi_{r} x_{r}+1-x_{r, r+1}\right) e(\boldsymbol{i}) & \text { if } i_{r}=i_{r+1}\end{cases} \\
& \varphi_{r}^{2} e(\boldsymbol{i})= \begin{cases}\left(1-x_{r, r+1}^{-2}\right) e(\boldsymbol{i}) & \text { if } i_{r} \neq i_{r+1}, \\
2 \varphi_{r} e(\boldsymbol{i}) & \text { if } i_{r}=i_{r+1}\end{cases} \\
& \varphi_{r} \varphi_{r+1} \varphi_{r} e(\boldsymbol{i})= \begin{cases}\left(\varphi_{r+1} \varphi_{r} \varphi_{r+1}+\varphi_{r}-\varphi_{r+1}\right) e(\boldsymbol{i}) & \text { if } i_{r}=i_{r+2}=i_{r+1}, \\
\left(\varphi_{r+1} \varphi_{r} \varphi_{r+1}+z_{r}\right) e(\boldsymbol{i}) & \text { if } i_{r}=i_{r+2} \neq i_{r+1}, \\
\varphi_{r+1} \varphi_{r} \varphi_{r+1} e(\boldsymbol{i}) & \text { otherwise, }\end{cases}
\end{aligned}
$$

where $z_{r}$ denotes $\left(x_{r, r+1}^{-1}-x_{r+1, r+2}^{-1}\right)\left(x_{r, r+1}^{-1} x_{r+1, r+2}^{-1}-x_{r, r+1}^{-1}-x_{r+1, r+2}^{-1}\right)$.

Proof. To see (3.14), it suffices to prove that its left hand side and right hand side act in the same way on $M_{j}$ for any finite dimensional $H_{\alpha}^{\Lambda}$-module $M$ and $\boldsymbol{j} \in I^{\alpha}$. If $j_{r}=j_{r+1}$ then $\varphi_{r} M_{\boldsymbol{j}} \subseteq M_{s_{r} \cdot \boldsymbol{j}}$ by (3.7). If $j_{r} \neq j_{r+1}$ then $\varphi_{r} e(\boldsymbol{j})=\theta_{r} x_{r, r+1}^{-1} e(\boldsymbol{j})$, hence $\varphi_{r} M_{\boldsymbol{j}}=\theta_{r} M_{\boldsymbol{j}}$ which is contained in $M_{s_{r} \cdot \boldsymbol{j}}$ by (3.12). Hence in any case $e\left(s_{r} \cdot \boldsymbol{i}\right) \varphi_{r}$ maps $M_{\boldsymbol{j}}$ to zero unless $\boldsymbol{j}=\boldsymbol{i}$, and it maps $v \in M_{\boldsymbol{i}}$ to $\varphi_{r} v$. This is the same as the action of $\varphi_{r} e(\boldsymbol{i})$, hence (3.14) is checked. The properties (3.15) and (3.16) are clear, and the properties (3.17) and (3.18) come easily from (3.12). For (3.19), if $i_{r}=i_{r+1}$ then we have that $\varphi_{r}^{2} e(\boldsymbol{i})=\left(s_{r}+1\right)^{2} e(\boldsymbol{i})=\left(2+2 s_{r}\right) e(\boldsymbol{i})=2 \varphi_{r} e(\boldsymbol{i})$. Now suppose that $i_{r} \neq i_{r+1}$. Then, using (3.12) and (3.11), we have

$$
\varphi_{r}^{2} e(\boldsymbol{i})=\theta_{r} x_{r, r+1}^{-1} \theta_{r} x_{r, r+1}^{-1} e(\boldsymbol{i})=-\theta_{r}^{2} x_{r, r+1}^{-2} e(\boldsymbol{i})=\left(1-x_{r, r+1}^{-2}\right) e(\boldsymbol{i}) .
$$

It remains to check (3.20). For this, let us stop writing $e(\boldsymbol{i})$ on the right of all expressions (but of course remember it is there). Assume without loss of generality that $r=1$ and denote $i:=i_{1}, j:=i_{2}, k:=i_{3}$. We consider five cases: 
Case 1: $i, j, k$ all distinct. Using (3.12), (3.13) and (3.14), we have:

$$
\begin{aligned}
\varphi_{2} \varphi_{1} \varphi_{2} & =\theta_{2} x_{2,3}^{-1} \theta_{1} x_{1,2}^{-1} \theta_{2} x_{2,3}^{-1}=\theta_{2} \theta_{1} \theta_{2} x_{1,2}^{-1} x_{1,3}^{-1} x_{2,3}^{-1} \\
& =\theta_{1} \theta_{2} \theta_{1} x_{2,3}^{-1} x_{1,3}^{-1} x_{1,2}^{-1}=\theta_{1} x_{1,2}^{-1} \theta_{2} x_{2,3}^{-1} \theta_{1} x_{1,2}^{-1}=\varphi_{1} \varphi_{2} \varphi_{1} .
\end{aligned}
$$

Case 2: $i=j \neq k$. Using (3.14), we see that $\varphi_{2} \varphi_{1} \varphi_{2}=\varphi_{1} \varphi_{2} \varphi_{1}$ is equivalent to $\left(s_{2}+1\right) \varphi_{1} \varphi_{2}=\varphi_{1} \varphi_{2}\left(s_{1}+1\right)$, or $s_{2} \varphi_{1} \varphi_{2}=\varphi_{1} \varphi_{2} s_{1}$. Also the relations in $H_{\alpha}^{\Lambda}$ give that $x_{1,3}^{-1} s_{1}=s_{1} x_{2,3}^{-1}+x_{2,3}^{-1} x_{1,3}^{-1}$ and $x_{2,3}^{-1} s_{1}=s_{1} x_{1,3}^{-1}-x_{2,3}^{-1} x_{1,3}^{-1}$ (remembering the idempotent $e(\boldsymbol{i})$ implicitly appears on the right so all the inverses here make sense). Now apply (3.12), (3.13) and these two relations to commute all $x$ 's to the right and show that both sides are equal to $s_{2} s_{1} s_{2}+$ $s_{2} s_{1} x_{2,3}^{-1}+s_{2} x_{2,3}^{-1} x_{1,3}^{-1}+x_{1,3}^{-1}$.

Case 3: $i \neq j=k$. This case is similar to Case 2 .

Case 4: $i=k \neq j$. Using (3.12), (3.13) and (3.14), we get

$$
\begin{aligned}
\varphi_{2} \varphi_{1} \varphi_{2}= & \theta_{2} x_{2,3}^{-1}\left(s_{1}+1\right) \theta_{2} x_{2,3}^{-1}=\theta_{2} x_{2,3}^{-1} s_{1} \theta_{2} x_{2,3}^{-1}-\theta_{2}^{2} x_{2,3}^{-2} \\
= & \theta_{2}\left(s_{1} x_{1,3}^{-1}-x_{2,3}^{-1} x_{1,3}^{-1}\right) \theta_{2} x_{2,3}^{-1}-\left(1-x_{2,3}^{2}\right) x_{2,3}^{-2} \\
= & \theta_{2} s_{1} \theta_{2} x_{1,2}^{-1} x_{2,3}^{-1}+\theta_{2}^{2} x_{2,3}^{-2} x_{1,2}^{-1}-x_{2,3}^{-2}+1 \\
= & \left(s_{2} x_{2,3}+1\right) s_{1} \theta_{2} x_{1,2}^{-1} x_{2,3}^{-1}+\left(1-x_{2,3}^{2}\right) x_{2,3}^{-2} x_{1,2}^{-1}-x_{2,3}^{-2}+1 \\
= & s_{2}\left(s_{1} x_{1,3}+1\right) \theta_{2} x_{1,2}^{-1} x_{2,3}^{-1}+s_{1} \theta_{2} x_{1,2}^{-1} x_{2,3}^{-1}+\left(x_{2,3}^{-2}-1\right)\left(x_{1,2}^{-1}-1\right) \\
= & s_{2} s_{1} \theta_{2} x_{2,3}^{-1}+s_{2} \theta_{2} x_{1,2}^{-1} x_{2,3}^{-1}+s_{1} \theta_{2} x_{1,2}^{-1} x_{2,3}^{-1}+\left(x_{2,3}^{-2}-1\right)\left(x_{1,2}^{-1}-1\right) \\
= & s_{2} s_{1}\left(s_{2} x_{2,3}+1\right) x_{2,3}^{-1}+s_{2}\left(s_{2} x_{2,3}+1\right) x_{1,2}^{-1} x_{2,3}^{-1} \\
& \quad+s_{1}\left(s_{2} x_{2,3}+1\right) x_{1,2}^{-1} x_{2,3}^{-1}+\left(x_{2,3}^{-2}-1\right)\left(x_{1,2}^{-1}-1\right) \\
= & s_{2} s_{1} s_{2}+s_{2} s_{1} x_{2,3}^{-1}+s_{1} s_{2} x_{1,2}^{-1}+s_{2} x_{1,2}^{-1} x_{2,3}^{-1}+s_{1} x_{1,2}^{-1} x_{2,3}^{-1} \\
& \quad+x_{1,2}^{-1}+\left(x_{2,3}^{-2}-1\right)\left(x_{1,2}^{-1}-1\right) .
\end{aligned}
$$

Similarly, we have

$$
\begin{gathered}
\varphi_{1} \varphi_{2} \varphi_{1}=s_{1} s_{2} s_{1}+s_{1} s_{2} x_{1,2}^{-1}+s_{2} s_{1} x_{2,3}^{-1}+s_{1} x_{2,3}^{-1} x_{1,2}^{-1}+s_{2} x_{2,3}^{-1} x_{1,2}^{-1} \\
+x_{2,3}^{-1}+\left(x_{1,2}^{-2}-1\right)\left(x_{2,3}^{-1}-1\right),
\end{gathered}
$$

and (3.20) now follows.

Case 5: $i=j=k$. This case follows since $\left(s_{1}+1\right)\left(s_{2}+1\right)\left(s_{1}+1\right)+s_{2}=$ $\left(s_{2}+1\right)\left(s_{1}+1\right)\left(s_{2}+1\right)+s_{1}$.

3.3. Khovanov-Lauda generators of $\boldsymbol{H}_{\boldsymbol{\alpha}}^{\boldsymbol{\Lambda}}$ in the degenerate case. For each $r=1, \ldots, d$, the elements

$$
y_{r}:=\sum_{i \in I^{\alpha}}\left(x_{r}-i_{r}\right) e(\boldsymbol{i})
$$

are nilpotent elements of the commutative algebra $P o l_{\alpha}^{\Lambda}$. So we are in the situation of (2.3) and get a homomorphism $F\left[\left[y_{1}, \ldots, y_{d}\right]\right] \rightarrow \operatorname{Pol}_{\alpha}^{\Lambda}$ mapping each $y_{r} \in F\left[\left[y_{1}, \ldots, y_{d}\right]\right]$ to the element (3.21). We are often going to abuse 
notation by using the same symbol for a power series $f \in F\left[\left[y_{1}, \ldots, y_{d}\right]\right]$ and for its image in $\mathrm{Pol}_{\alpha}^{\Lambda}$ under this homomorphism.

For $1 \leq r<d$ and $\boldsymbol{i} \in I^{\alpha}$, we define power series $p_{r}(\boldsymbol{i}) \in F\left[\left[y_{r}, y_{r+1}\right]\right]$ by setting

$$
p_{r}(\boldsymbol{i}):= \begin{cases}1 & \text { if } i_{r}=i_{r+1}, \\ \left(i_{r}-i_{r+1}+y_{r}-y_{r+1}\right)^{-1} & \text { if } i_{r} \neq i_{r+1}\end{cases}
$$

The following facts are easy to check:

$$
\begin{aligned}
{ }^{s_{r}} p_{r}\left(s_{r} \cdot \boldsymbol{i}\right) & =-p_{r}(\boldsymbol{i}) & & \text { if } i_{r} \neq i_{r+1} ; \\
{ }^{s_{r}} p_{r+1}\left(s_{r} \cdot \boldsymbol{i}\right) & ={ }^{s_{r+1}} p_{r}\left(s_{r+1} \cdot \boldsymbol{i}\right) & & \text { for any } \boldsymbol{i} .
\end{aligned}
$$

Note also for all $1 \leq r<d$ and $i \in I^{\alpha}$ that

$$
\begin{aligned}
p_{r}(\boldsymbol{i}) e(\boldsymbol{i}) & = \begin{cases}e(\boldsymbol{i}) & \text { if } i_{r}=i_{r+1}, \\
x_{r, r+1}^{-1} e(\boldsymbol{i}) & \text { if } i_{r} \neq i_{r+1}\end{cases} \\
\varphi_{r} & =\sum_{\boldsymbol{i} \in I^{\alpha}}\left(s_{r}+p_{r}(\boldsymbol{i})\right) e(\boldsymbol{i}) .
\end{aligned}
$$

Also make an arbitrary but henceforth fixed choice of (invertible) elements $q_{r}(\boldsymbol{i}) \in F\left[\left[y_{r}, y_{r+1}\right]\right]$ with the following properties:

$$
\begin{aligned}
& q_{r}(\boldsymbol{i})=1+y_{r+1}-y_{r} \quad \text { if } i_{r}=i_{r+1} ; \\
& q_{r}(\boldsymbol{i})^{s_{r}} q_{r}\left(s_{r} \cdot \boldsymbol{i}\right)=\left\{\begin{array}{ll}
1-p_{r}(\boldsymbol{i})^{2} & \text { if } i_{r}+i_{r+1}, \\
\left(1-p_{r}(\boldsymbol{i})^{2}\right) /\left(y_{r+1}-y_{r}\right) & \text { if } i_{r} \rightarrow i_{r+1}, \\
\left(1-p_{r}(\boldsymbol{i})^{2}\right) /\left(y_{r}-y_{r+1}\right) & \text { if } i_{r} \leftarrow i_{r+1}, \\
\frac{1-p_{r}(\boldsymbol{i})^{2}}{\left(y_{r+1}-y_{r}\right)\left(y_{r}-y_{r+1}\right)} & \text { if } i_{r} \rightleftarrows i_{r+1}
\end{array} ;,\right. \\
& { }^{s_{r}} q_{r+1}\left(s_{r+1} s_{r} \cdot \boldsymbol{i}\right)={ }^{s_{r+1}} q_{r}\left(s_{r} s_{r+1} \cdot \boldsymbol{i}\right) \quad \text { for any } \boldsymbol{i} .
\end{aligned}
$$

Note in the fractions on the right hand side of (3.28) that the numerator is divisible by the denominator in $F\left[\left[y_{r}, y_{r+1}\right]\right]$, so this makes sense. Moreover, it is always possible to choose such power series $q_{r}(\boldsymbol{i})$. For instance, one could take

$$
q_{r}(\boldsymbol{i}):= \begin{cases}1+y_{r+1}-y_{r} & \text { if } i_{r}=i_{r+1}, \\ 1-p_{r}(\boldsymbol{i}) & \text { if } i_{r}+i_{r+1}, \\ \left(1-p_{r}(\boldsymbol{i})^{2}\right) /\left(y_{r+1}-y_{r}\right) & \text { if } i_{r} \rightarrow i_{r+1}, \\ 1 & \text { if } i_{r} \leftarrow i_{r+1}, \\ \left(1-p_{r}(\boldsymbol{i})\right) /\left(y_{r+1}-y_{r}\right) & \text { if } i_{r} \rightleftarrows i_{r+1},\end{cases}
$$

although we do not want to restrict ourselves to this particular choice.

Now, the Khovanov-Lauda generators of $H_{\alpha}^{\Lambda}$ are the elements

$$
\left\{e(\boldsymbol{i}) \mid \boldsymbol{i} \in I^{\alpha}\right\} \cup\left\{y_{1}, \ldots, y_{d}\right\} \cup\left\{\psi_{1}, \ldots, \psi_{d-1}\right\},
$$

where $y_{r}$ is the element defined by (3.21) and

$$
\psi_{r}:=\sum_{\boldsymbol{i} \in I^{\alpha}} \varphi_{r} q_{r}(\boldsymbol{i})^{-1} e(\boldsymbol{i})=\sum_{\boldsymbol{i} \in I^{\alpha}}\left(s_{r}+p_{r}(\boldsymbol{i})\right) q_{r}(\boldsymbol{i})^{-1} e(\boldsymbol{i}) .
$$

Theorem 3.2. The elements (3.31) of $H_{\alpha}^{\Lambda}$ satisfy the defining relations (1.6) -(1.15) of the cyclotomic Khovanov-Lauda algebra. 
Proof. We have by (1.2) that $\prod_{i \in I}\left(x_{1}-i\right)^{\left(\Lambda, \alpha_{i}\right)}=0$. Moreover, if $i \neq i_{1}$ then $\left(x_{1}-i\right) e(\boldsymbol{i})$ is invertible in $\operatorname{Pol}_{\alpha}^{\Lambda} e(\boldsymbol{i})$. So $\left(x_{1}-i_{1}\right)^{\left(\Lambda, \alpha_{i_{1}}\right)} e(\boldsymbol{i})=0$, which immediately implies (1.6). We already know that (1.7) holds. The relations (1.8), (1.9), (1.10) and (1.11) follow using the fact that $P o l_{\alpha}^{\Lambda}$ is commutative and the properties (3.14), (3.15) and (3.16) of the intertwining elements.

For (1.13), in view of (1.8), we have

$$
y_{r+1} \psi_{r} e(\boldsymbol{i})=y_{r+1} e\left(s_{r} \cdot \boldsymbol{i}\right) \psi_{r} e(\boldsymbol{i})=\left(x_{r+1}-i_{r}\right) \varphi_{r} q_{r}(\boldsymbol{i})^{-1} e(\boldsymbol{i}) .
$$

If $i_{r} \neq i_{r+1}$, this equals $\varphi_{r} q_{r}(\boldsymbol{i})^{-1}\left(x_{r}-i_{r}\right) e(\boldsymbol{i})=\psi_{r} y_{r} e(\boldsymbol{i})$ by (3.18). If $i_{r}=i_{r+1}$, then (3.33) gives

$$
\begin{aligned}
y_{r+1} \psi_{r} e(\boldsymbol{i}) & =\left(x_{r+1}-i_{r}\right)\left(s_{r}+1\right)\left(1-x_{r, r+1}\right)^{-1} e(\boldsymbol{i}) \\
& =\left(\left(s_{r}+1\right)\left(x_{r}-i_{r}\right)+1-x_{r, r+1}\right)\left(1-x_{r, r+1}\right)^{-1} e(\boldsymbol{i}) \\
& =\left(\psi_{r} y_{r}+1\right) e(\boldsymbol{i}) .
\end{aligned}
$$

The proof of (1.12) is similar.

For (1.14), we have that

$$
\psi_{r}^{2} e(\boldsymbol{i})=\varphi_{r} q_{r}\left(s_{r} \cdot \boldsymbol{i}\right)^{-1} \psi_{r} e(\boldsymbol{i}) .
$$

If $i_{r}=i_{r+1}$, the relations in $H_{\alpha}^{\Lambda}$ give easily that $\left(s_{r}+1\right)\left(1+x_{r, r+1}\right)=$ $\left(1-x_{r, r+1}\right)\left(s_{r}-1\right)$, hence we get from (3.34) that

$$
\begin{aligned}
\psi_{r}^{2} e(\boldsymbol{i}) & =\left(s_{r}+1\right)\left(1-x_{r, r+1}\right)^{-1}\left(s_{r}+1\right)\left(1-x_{r, r+1}\right)^{-1} e(\boldsymbol{i}) \\
& =\left(s_{r}+1\right)\left(s_{r}-1\right)\left(1+x_{r, r+1}\right)^{-1}\left(1-x_{r, r+1}\right)^{-1} e(\boldsymbol{i})=0 .
\end{aligned}
$$

Now suppose that $i_{r} \neq i_{r+1}$. Note as we have now checked the relations (1.10), (1.12) and (1.13), the identity (2.6) holds in the present situation. Using (2.6), (3.19) and (3.25), the equation (3.34) becomes

$$
\begin{aligned}
\psi_{r}^{2} e(\boldsymbol{i}) & =\varphi_{r} \psi_{r}\left({ }^{s_{r}} q_{r}\left(s_{r} \cdot \boldsymbol{i}\right)\right)^{-1} e(\boldsymbol{i})=\varphi_{r}^{2} q_{r}(\boldsymbol{i})^{-1}\left({ }^{s_{r}} q_{r}\left(s_{r} \cdot \boldsymbol{i}\right)\right)^{-1} e(\boldsymbol{i}) \\
& =\left(1-p_{r}(\boldsymbol{i})^{2}\right)\left(q_{r}(\boldsymbol{i})^{s_{r}} q_{r}\left(s_{r} \cdot \boldsymbol{i}\right)\right)^{-1} e(\boldsymbol{i}) .
\end{aligned}
$$

Using (3.28), this simplifies to give the right hand side of (1.14).

Finally we prove (1.15). Let us stop writing $e(\boldsymbol{i})$ at the right of all expressions. Assume without loss of generality that $r=1, d=3$, and denote $i:=i_{1}, j:=i_{2}, k:=i_{3}$.

Case 1: $i, j, k$ all distinct. Using (2.6) and (1.8), we get

$$
\begin{aligned}
\psi_{1} \psi_{2} \psi_{1} & =\varphi_{1} q_{1}(j k i)^{-1} \psi_{2} \psi_{1}=\varphi_{1} \psi_{2} \psi_{1}\left({ }^{s_{1} s_{2}} q_{1}(j k i)\right)^{-1} \\
& =\varphi_{1} \varphi_{2} q_{2}(j i k)^{-1} \psi_{1}\left({ }^{s_{1} s_{2}} q_{1}(j k i)\right)^{-1} \\
& =\varphi_{1} \varphi_{2} \psi_{1}\left({ }^{s_{1}} q_{2}(j i k)^{s_{1} s_{2}} q_{1}(j k i)\right)^{-1} \\
& =\varphi_{1} \varphi_{2} \varphi_{1}\left(q_{1}(i j k)^{s_{1}} q_{2}(j i k)^{s_{1} s_{2}} q_{1}(j k i)\right)^{-1} .
\end{aligned}
$$

Similarly,

$$
\psi_{2} \psi_{1} \psi_{2}=\varphi_{2} \varphi_{1} \varphi_{2}\left(q_{2}(i j k)^{s_{2}} q_{1}(i k j)^{s_{2} s_{1}} q_{2}(k i j)\right)^{-1} .
$$

Now, $\varphi_{1} \varphi_{2} \varphi_{1}=\varphi_{2} \varphi_{1} \varphi_{2}$ by (3.20), and also $q_{2}(i j k)={ }^{s_{1} s_{2}} q_{1}(j k i),{ }^{s_{2}} q_{1}(i k j)=$ ${ }^{s_{1}} q_{2}(j i k)$ and ${ }^{s_{2} s_{1}} q_{2}(k i j)=q_{1}(i j k)$ by (3.29). Thus (1.15) holds. 
Case 2: $i=j \neq k$. As in the previous case, we get that

$$
\psi_{2} \psi_{1} \psi_{2}=\varphi_{2} \varphi_{1} \varphi_{2}\left(q_{2}(i i k)^{s_{2}} q_{1}(i k i)^{s_{2} s_{1}} q_{2}(k i i)\right)^{-1} .
$$

On the other hand, by (2.6) and (3.29),

$$
\begin{aligned}
\psi_{1} \psi_{2} \psi_{1}= & \varphi_{1} q_{1}(i k i)^{-1} \psi_{2} \psi_{1} \\
= & \varphi_{1} \psi_{2} \psi_{1}\left({ }^{s_{1} s_{2}} q_{1}(i k i)\right)^{-1}+\varphi_{1} \psi_{2} \partial_{1}\left(\left({ }^{s_{2}} q_{1}(i k i)\right)^{-1}\right) \\
= & \varphi_{1} \varphi_{2} q_{2}(i i k)^{-1} \psi_{1}\left({ }^{s_{1} s_{2}} q_{1}(i k i)\right)^{-1}+\varphi_{1} \varphi_{2} q_{2}(i i k)^{-1} \partial_{1}\left(\left({ }^{s_{2}} q_{1}(i k i)\right)^{-1}\right) \\
= & \varphi_{1} \varphi_{2} \psi_{1}\left({ }^{s_{1}} q_{2}(i i k)^{s_{1} s_{2}} q_{1}(i k i)\right)^{-1}+\varphi_{1} \varphi_{2} \partial_{1}\left(q_{2}(i i k)^{-1}\right)\left({ }^{s_{1} s_{2}} q_{1}(i k i)\right)^{-1} \\
& \quad+\varphi_{1} \varphi_{2} q_{2}(i i k)^{-1} \partial_{1}\left(\left({ }^{s_{2}} q_{1}(i k i)\right)^{-1}\right) . \\
= & \varphi_{1} \varphi_{2} \varphi_{1}\left(q_{1}(i i k)^{s_{1}} q_{2}(i i k)^{s_{1} s_{2}} q_{1}(i k i)\right)^{-1} \\
& \quad+\varphi_{1} \varphi_{2} q_{2}(i i k)^{-1} \partial_{1}\left(q_{2}(i i k)^{-1}+\left({ }^{s_{1}} q_{2}(i i k)\right)^{-1}\right) .
\end{aligned}
$$

The first term of the last expression equals the right hand side of (3.35) by (3.20) and (3.29). It remains to observe that the second term of the last expression is zero, as $\partial_{1} f=0$ for any $f$ with ${ }^{s_{1}} f=f$.

Case 3: $i \neq j=k$. This case is similar to Case 2 .

Case 4: $i=k \neq j$. As in the previous cases we compute:

$$
\begin{gathered}
\psi_{1} \psi_{2} \psi_{1}=\varphi_{1} \varphi_{2} \varphi_{1}\left(q_{1}(i j i)^{s_{1}} q_{2}(j i i)^{s_{1} s_{2}} q_{1}(j i i)\right)^{-1} \\
+\left(1-x_{1,2}^{-2}\right) q_{1}(i j i)^{-1}\left({ }^{s_{1}} \partial_{2}\left(q_{1}(j i i)^{-1}\right)\right), \\
\psi_{2} \psi_{1} \psi_{2}=\varphi_{2} \varphi_{1} \varphi_{2}\left(q_{2}(i j i)^{s_{2}} q_{1}(i i j)^{s_{2} s_{1}} q_{2}(i i j)\right)^{-1} \\
+\left(1-x_{2,3}^{-2}\right) q_{2}(i j i)^{-1}\left({ }^{s_{2}} \partial_{1}\left(q_{2}(i i j)^{-1}\right)\right) .
\end{gathered}
$$

So by (3.20), (3.29), and (3.25), we get that $\psi_{1} \psi_{2} \psi_{1}-\psi_{2} \psi_{1} \psi_{2}=A+B-C$, where

$$
\begin{gathered}
A=\left(p_{1}(i j i)-p_{2}(i j i)\right)\left(p_{1}(i j i) p_{2}(i j i)-p_{1}(i j i)-p_{2}(i j i)\right) \\
\quad \times\left(q_{1}(i j i)^{s_{1}} q_{2}(j i i) q_{2}(i j i)\right)^{-1}, \\
B=\left(1-p_{1}(i j i)^{2}\right) q_{1}(i j i)^{-1}\left({ }^{s_{1}} \partial_{2}\left(q_{1}(j i i)^{-1}\right)\right) \\
C=\left(1-p_{2}(i j i)^{2}\right) q_{2}(i j i)^{-1}\left({ }^{s_{2}} \partial_{1}\left(q_{2}(i i j)^{-1}\right)\right) .
\end{gathered}
$$

By substituting $p_{1}(i j i)=\left(i-j+y_{1}-y_{2}\right)^{-1}, p_{2}(i j i)=\left(j-i+y_{2}-y_{3}\right)^{-1}$ and putting over a common denominator, it is straightforward to check the following power series identity:

$$
p_{1}(i j i)+p_{2}(i j i)=\left(y_{1}-y_{3}\right) p_{1}(i j i) p_{2}(i j i) .
$$

Hence:

$$
\frac{p_{1}(i j i) p_{2}(i j i)-p_{1}(i j i)-p_{2}(i j i)}{1+y_{3}-y_{1}}=\frac{p_{1}(i j i)+p_{2}(i j i)}{y_{1}-y_{3}} .
$$

Using this and noting ${ }^{{ }_{1}} q_{2}(j i i)=1+y_{3}-y_{1}$, we deduce that

$$
A=\frac{\left(p_{1}(i j i)^{2}-p_{2}(i j i)^{2}\right) q_{1}(i j i)^{-1} q_{2}(i j i)^{-1}}{y_{1}-y_{3}} .
$$


Using the definition (2.1) and the property (3.28), we have that

$$
{ }^{s_{1}} \partial_{2}\left(q_{1}(j i i)^{-1}\right)=\frac{q_{2}(i j i)^{-1}-\left({ }^{s_{1}} q_{1}(j i i)\right)^{-1}}{y_{1}-y_{3}} .
$$

Substituting this into $B$, we get that

$$
B=\frac{\left(1-p_{1}(i j i)^{2}\right)\left(\left(q_{1}(i j i)^{-1} q_{2}(i j i)^{-1}-\left(q_{1}(i j i)^{s_{1}} q_{1}(j i i)\right)^{-1}\right)\right.}{y_{1}-y_{3}} .
$$

Similarly,

$$
C=\frac{\left(1-p_{2}(i j i)^{2}\right)\left(q_{1}(i j i)^{-1} q_{2}(i j i)^{-1}-\left(q_{2}(i j i)^{s_{2}} q_{2}(i i j)\right)^{-1}\right)}{y_{1}-y_{3}} .
$$

The equations (3.37), (3.38) and (3.39) easily give that $A+B-C$ equals

$$
\frac{\left(1-p_{2}(i j i)^{2}\right)\left(q_{2}(i j i)^{s_{2}} q_{2}(i i j)\right)^{-1}-\left(1-p_{1}(i j i)^{2}\right)\left(q_{1}(i j i)^{s_{1}} q_{1}(j i i)\right)^{-1}}{y_{1}-y_{3}} .
$$

Finally by (3.28) this is 0 if $i+j, 1$ if $i \rightarrow j,-1$ if $i \leftarrow j$ or $-2 y_{2}+y_{1}+y_{3}$ if $i \rightleftarrows j$. This imples (1.15).

Case 5: $i=j=k$. We leave this case as an exercise to the reader.

3.4. Degenerate Hecke generators of $\boldsymbol{R}_{\boldsymbol{\alpha}}^{\boldsymbol{\Lambda}}$. Let $R_{\alpha}^{\Lambda}$ be the cyclotomic Khovanov-Lauda algebra from $\$ 2.2$. Using the homomorphism (2.5), we can regard the power series $p_{r}(\boldsymbol{i})$ from $(3.22)$ as elements of $R_{\alpha}^{\Lambda}$. Similarly, the power series $q_{r}(\boldsymbol{i})$ satisfying (3.27)-(3.29) that were chosen in 33.3 give rise to elements $q_{r}(\boldsymbol{i}) \in R_{\alpha}^{\Lambda}$. The degenerate Hecke generators of $R_{\alpha}^{\Lambda}$ are the elements

$$
\left\{x_{1}, \ldots, x_{d}\right\} \cup\left\{s_{1}, \ldots, s_{d-1}\right\}
$$

where

$$
\begin{aligned}
x_{r} & :=\sum_{\boldsymbol{i} \in I^{\alpha}}\left(y_{r}+i_{r}\right) e(\boldsymbol{i}), \\
s_{r} & :=\sum_{\boldsymbol{i} \in I^{\alpha}}\left(\psi_{r} q_{r}(\boldsymbol{i})-p_{r}(\boldsymbol{i})\right) e(\boldsymbol{i}) .
\end{aligned}
$$

We note by (1.14) and (3.28) that

$$
\psi_{r}^{2} q_{r}(\boldsymbol{i})^{s_{r}} q_{r}\left(s_{r} \cdot \boldsymbol{i}\right) e(\boldsymbol{i})=\left(1-p_{r}(\boldsymbol{i})^{2}\right) e(\boldsymbol{i})
$$

for all $i \in I^{\alpha}$ and $1 \leq r<d$. The following result is the key technical result needed to complete the proof of our Main Theorem in the degenerate case.

Theorem 3.3. The elements (3.40) of $R_{\alpha}^{\Lambda}$ satisfy the defining relations (3.2) - (3.5) of the degenerate affine Hecke algebra $H_{d}$.

Proof. The polynomial relation (3.2) is obvious: the $x_{r}$ 's commute because the $y_{r}$ 's and $e(\boldsymbol{i})$ 's do. The mixed relation (3.3) is clear for $s \neq r, r+1$. For the remaining mixed relation, it suffices to show that

$$
\left(s_{r} x_{r+1}-x_{r} s_{r}\right) e(\boldsymbol{i})=e(\boldsymbol{i})
$$


for every $i \in I^{\alpha}$. Expand the definitions (3.41)-(3.42) using (1.8) gives:

$$
\begin{aligned}
s_{r} x_{r+1} e(\boldsymbol{i}) & =\left(\psi_{r} q_{r}(\boldsymbol{i})-p_{r}(\boldsymbol{i})\right)\left(y_{r+1}+i_{r+1}\right) e(\boldsymbol{i}), \\
x_{r} s_{r} e(\boldsymbol{i}) & =x_{r}\left(\psi_{r} q_{r}(\boldsymbol{i})-p_{r}(\boldsymbol{i})\right) e(\boldsymbol{i}) \\
& =x_{r} e\left(s_{r} \cdot \boldsymbol{i}\right) \psi_{r} q_{r}(\boldsymbol{i}) e(\boldsymbol{i})-x_{r} e(\boldsymbol{i}) p_{r}(\boldsymbol{i}) e(\boldsymbol{i}) \\
& =\left(y_{r}+i_{r+1}\right) \psi_{r} q_{r}(\boldsymbol{i}) e(\boldsymbol{i})-\left(y_{r}+i_{r}\right) p_{r}(\boldsymbol{i}) e(\boldsymbol{i}) .
\end{aligned}
$$

Hence

$\left(s_{r} x_{r+1}-x_{r} s_{r}\right) e(\boldsymbol{i})=\left(i_{r}-i_{r+1}+y_{r}-y_{r+1}\right) p_{r}(\boldsymbol{i}) e(\boldsymbol{i})+\left(\psi_{r} y_{r+1}-y_{r} \psi_{r}\right) q_{r}(\boldsymbol{i}) e(\boldsymbol{i})$.

Applying (1.12), (3.22) and (3.27), we have that $\left(\psi_{r} y_{r+1}-y_{r} \psi_{r}\right) e(\boldsymbol{i})=e(\boldsymbol{i})$, $p_{r}(\boldsymbol{i})=1$ and $q_{r}(\boldsymbol{i})=1+y_{r+1}-y_{r}$ if $i_{r}=i_{r+1}$, or $\left(\psi_{r} y_{r+1}-y_{r} \psi_{r}\right) e(\boldsymbol{i})=0$ and $p_{r}(\boldsymbol{i})=\left(i_{r}-i_{r+1}+y_{r}-y_{r+1}\right)^{-1}$ if $i_{r} \neq i_{r+1}$. Making these substitutions gives easily that $\left(s_{r} x_{r+1}-x_{r} s_{r}\right) e(\boldsymbol{i})=e(\boldsymbol{i})$ as required.

Next we check the quadratic relation (3.4). For this we need to show that

$$
s_{r}^{2} e(\boldsymbol{i})=e(\boldsymbol{i})
$$

for each $i \in I^{\alpha}$. Expanding the definition (3.42), we get that

$$
\begin{aligned}
s_{r}^{2} e(\boldsymbol{i}) & =s_{r}\left(\psi_{r} q_{r}(\boldsymbol{i})-p_{r}(\boldsymbol{i})\right) e(\boldsymbol{i})=s_{r} e\left(s_{r} \cdot \boldsymbol{i}\right) \psi_{r} q_{r}(\boldsymbol{i}) e(\boldsymbol{i})-s_{r} e(\boldsymbol{i}) p_{r}(\boldsymbol{i}) e(\boldsymbol{i}) \\
& =\left(\psi_{r} q_{r}\left(s_{r} \cdot \boldsymbol{i}\right)-p_{r}\left(s_{r} \cdot \boldsymbol{i}\right)\right) \psi_{r} q_{r}(\boldsymbol{i}) e(\boldsymbol{i})-\left(\psi_{r} q_{r}(\boldsymbol{i})-p_{r}(\boldsymbol{i})\right) p_{r}(\boldsymbol{i}) e(\boldsymbol{i}) \\
& =\left(\psi_{r} q_{r}\left(s_{r} \cdot \boldsymbol{i}\right) \psi_{r} q_{r}(\boldsymbol{i})-p_{r}\left(s_{r} \cdot \boldsymbol{i}\right) \psi_{r} q_{r}(\boldsymbol{i})-\psi_{r} q_{r}(\boldsymbol{i}) p_{r}(\boldsymbol{i})+p_{r}(\boldsymbol{i})^{2}\right) e(\boldsymbol{i}) .
\end{aligned}
$$

If $i_{r}=i_{r+1}$, we use (3.22) and (3.27) to get from this that

$$
s_{r}^{2} e(\boldsymbol{i})=\left(\psi_{r}\left(1+y_{r+1}-y_{r}\right) \psi_{r} q_{r}(\boldsymbol{i})-2 q_{r}(\boldsymbol{i})+1\right) e(\boldsymbol{i}) .
$$

Using (1.12)-(1.13) to commute $y$ 's to the right and noting that $\psi_{r}^{2} e(\boldsymbol{i})=0$ by (1.14), this easily simplifies to give the desired equation $s_{r}^{2} e(\boldsymbol{i})=e(\boldsymbol{i})$. Instead, if $i_{r} \neq i_{r+1}$, then we again commute $y$ 's to the right and use (3.43) and $(3.23)$ to get that

$$
s_{r}^{2} e(\boldsymbol{i})=\left(\left(1-p_{r}(\boldsymbol{i})^{2}\right)+\psi_{r} p_{r}(\boldsymbol{i}) q_{r}(\boldsymbol{i})-\psi_{r} q_{r}(\boldsymbol{i}) p_{r}(\boldsymbol{i})+p_{r}(\boldsymbol{i})^{2}\right) e(\boldsymbol{i})=e(\boldsymbol{i}) .
$$

This completes the proof of (3.4).

Finally we need to check the braid relations (3.5). The commuting braid relation is obvious. For the length three braid relation, we assume without loss of generality that $r=1$ and $d=3$, and need to show that

$$
s_{2} s_{1} s_{2} e(i j k)=s_{1} s_{2} s_{1} e(i j k)
$$

for all $i, j, k$. To simplify notation for the remainder of the proof, we stop writing $e(i j k)$ on the right hand side of all expressions, but remember it is always there. Expanding the definition (3.42) like in the previous paragraph, $s_{2} s_{1} s_{2}$ and $s_{1} s_{2} s_{1}$ equal

$$
\begin{aligned}
& -p_{2}(i j k) p_{1}(i j k) p_{2}(i j k)+\psi_{2} q_{2}(i j k) p_{1}(i j k) p_{2}(i j k) \\
& +p_{2}(j i k) \psi_{1} q_{1}(i j k) p_{2}(i j k)-\psi_{2} q_{2}(j i k) \psi_{1} q_{1}(i j k) p_{2}(i j k) \\
& +p_{2}(i k j) p_{1}(i k j) \psi_{2} q_{2}(i j k)-\psi_{2} q_{2}(i k j) p_{1}(i k j) \psi_{2} q_{2}(i j k) \\
& -p_{2}(k i j) \psi_{1} q_{1}(i k j) \psi_{2} q_{2}(i j k)+\psi_{2} q_{2}(k i j) \psi_{1} q_{1}(i k j) \psi_{2} q_{2}(i j k),
\end{aligned}
$$


and

$$
\begin{aligned}
& -p_{1}(i j k) p_{2}(i j k) p_{1}(i j k)+\psi_{1} q_{1}(i j k) p_{2}(i j k) p_{1}(i j k) \\
& +p_{1}(i k j) \psi_{2} q_{2}(i j k) p_{1}(i j k)-\psi_{1} q_{1}(i k j) \psi_{2} q_{2}(i j k) p_{1}(i j k) \\
& +p_{1}(j i k) p_{2}(j i k) \psi_{1} q_{1}(i j k)-\psi_{1} q_{1}(j i k) p_{2}(j i k) \psi_{1} q_{1}(i j k) \\
& -p_{1}(j k i) \psi_{2} q_{2}(j i k) \psi_{1} q_{1}(i j k)+\psi_{1} q_{1}(j k i) \psi_{2} q_{2}(j i k) \psi_{1} q_{1}(i j k),
\end{aligned}
$$

respectively. We have to prove that (3.44) equals (3.45). For this we consider five cases. The strategy is always to commute all $\psi$ 's to the left using (2.6) then to compare various $\psi$-coefficients.

Case 1: $i, j, k$ are all different. By (2.6), (3.44) equals

$$
\begin{aligned}
& -p_{2}(i j k) p_{1}(i j k) p_{2}(i j k)+\psi_{2} q_{2}(i j k) p_{1}(i j k) p_{2}(i j k) \\
& +\psi_{1}{ }^{s_{1}} p_{2}(j i k) q_{1}(i j k) p_{2}(i j k)-\psi_{2} \psi_{1}^{s_{1}} q_{2}(j i k) q_{1}(i j k) p_{2}(i j k) \\
& +\psi_{2}{ }^{s_{2}} p_{2}(i k j)^{s_{2}} p_{1}(i k j) q_{2}(i j k)-\left(\psi_{2}^{2}\right)^{s_{2}} q_{2}(i k j)^{s_{2}} p_{1}(i k j) q_{2}(i j k) \\
& -\psi_{1} \psi_{2}{ }^{s^{s_{1}}} p_{2}(k i j)^{s_{2}} q_{1}(i k j) q_{2}(i j k)+\psi_{2} \psi_{1} \psi_{2}{ }^{s_{2} s_{1}} q_{2}(k i j)^{s_{2}} q_{1}(i k j) q_{2}(i j k),
\end{aligned}
$$

and (3.45) equals

$$
\begin{aligned}
& -p_{1}(i j k) p_{2}(i j k) p_{1}(i j k)+\psi_{1} q_{1}(i j k) p_{2}(i j k) p_{1}(i j k) \\
& +\psi_{2}{ }^{s_{2}} p_{1}(i k j) q_{2}(i j k) p_{1}(i j k)-\psi_{1} \psi_{2}{ }^{s_{2}} q_{1}(i k j) q_{2}(i j k) p_{1}(i j k) \\
& +\psi_{1}{ }^{s_{1}} p_{1}(j i k)^{s_{1}} p_{2}(j i k) q_{1}(i j k)-\left(\psi_{1}^{2}\right)^{s_{1}} q_{1}(j i k)^{s_{1}} p_{2}(j i k) q_{1}(i j k) \\
& -\psi_{2} \psi_{1}^{s_{1} s_{2}} p_{1}(j k i)^{s_{1}} q_{2}(j i k) q_{1}(i j k)+\psi_{1} \psi_{2} \psi_{1}^{{ }^{s_{1}} s_{2}} q_{1}(j k i)^{s_{1}} q_{2}(j i k) q_{1}(i j k) .
\end{aligned}
$$

Note that $\psi_{2} \psi_{1} \psi_{2}=\psi_{1} \psi_{2} \psi_{1}$ under our assumptions on $i, j, k$, and the corresponding coefficients are equal to each other by (3.29). For the $\psi_{1} \psi_{2^{-}}$ coefficients, we need to observe by (3.24) that ${ }^{s_{2} s_{1}} p_{2}(k i j)=p_{1}(i j k)$. The $\psi_{2} \psi_{1}$-coefficients are treated similarly. For the $\psi_{1}$-coefficients, it suffices to prove that

$$
{ }^{s_{1}} p_{2}(j i k) p_{2}(i j k)=p_{2}(i j k) p_{1}(i j k)+{ }^{s_{1}} p_{1}(j i k){ }^{s_{1}} p_{2}(j i k),
$$

which is easily checked by expanding the definition (3.22) and clearing denominators. The $\psi_{2}$-coefficients are handled similarly. Finally, the constant term reduces using (3.43) and the observation that ${ }^{s_{2}} p_{1}(i k j)={ }^{s_{1}} p_{2}(j i k)$ to checking that

$$
\left({ }^{s_{2}} p_{1}(i k j)-p_{1}(i j k)\right) p_{2}(i j k)^{2}=\left({ }^{s_{1}} p_{2}(j i k)-p_{2}(i j k)\right) p_{1}(i j k)^{2} .
$$

Again this identity follows by an explicit expansion using (3.22).

Case 2: $i=j \neq k$. We have $p_{1}(i i k)=p_{2}(k i i)=1$ by definition. So, using (2.6), the expression (3.44) equals

$$
\begin{aligned}
& -p_{2}(i i k)^{2}+\psi_{2} q_{2}(i i k) p_{2}(i i k)+\psi_{1}^{s_{1}} p_{2}(i i k) q_{1}(i i k) p_{2}(i i k) \\
& +\partial_{1}\left(p_{2}(i i k)\right) q_{1}(i i k) p_{2}(i i k)-\psi_{2} \psi_{1}^{s_{1}} q_{2}(i i k) q_{1}(i i k) p_{2}(i i k) \\
& -\psi_{2} \partial_{1}\left(q_{2}(i i k)\right) q_{1}(i i k) p_{2}(i i k)+\psi_{2}{ }^{s_{2}} p_{2}(i k i)^{s_{2}} p_{1}(i k i) q_{2}(i i k) \\
& -\left(\psi_{2}^{2}\right)^{s_{2}} q_{2}(i k i)^{s_{2}} p_{1}(i k i) q_{2}(i i k)-\psi_{1} \psi_{2}{ }^{s_{2}} q_{1}(i k i) q_{2}(i i k) \\
& +\psi_{2} \psi_{1} \psi_{2}^{s_{2} s_{1}} q_{2}(k i i)^{s_{2}} q_{1}(i k i) q_{2}(i i k)
\end{aligned}
$$


Similarly, using also (2.2), (3.45) becomes

$$
\begin{aligned}
& -p_{2}(i i k)+\psi_{1} q_{1}(i i k) p_{2}(i i k)+\psi_{2}{ }^{s_{2}} p_{1}(i k i) q_{2}(i i k)-\psi_{1} \psi_{2}{ }^{s_{2}} q_{1}(i k i) q_{2}(i i k) \\
& +\psi_{1}{ }^{s_{1}} p_{2}(i i k) q_{1}(i i k)+\partial_{1}\left(p_{2}(i i k)\right) q_{1}(i i k)-\left(\psi_{1}^{2}\right)^{s_{1}} q_{1}(i i k)^{s_{1}} p_{2}(i i k) q_{1}(i i k) \\
& -\psi_{1} \partial_{1}\left(q_{1}(i i k)\right)^{s_{1}} p_{2}(i i k) q_{1}(i i k)-\psi_{1} \partial_{1}\left(p_{2}(i i k)\right) q_{1}(i i k)^{2} \\
& -\psi_{2} \psi_{1}{ }^{s_{1} s_{2}} p_{1}(i k i)^{s_{1}} q_{2}(i i k) q_{1}(i i k)-\psi_{2} \partial_{1}\left({ }^{s_{2}} p_{1}(i k i)\right)^{s_{1}} q_{2}(i i k) q_{1}(i i k) \\
& -\psi_{2}{ }^{s_{2}} p_{1}(i k i) \partial_{1}\left(q_{2}(i i k)\right) q_{1}(i i k)+\psi_{1} \psi_{2} \psi_{1}^{s_{1} s_{2}} q_{1}(i k i)^{s_{1}} q_{2}(i i k) q_{1}(i i k) \\
& +\psi_{1} \psi_{2} \partial_{1}\left({ }^{s_{2}} q_{1}(i k i)\right)^{s_{1}} q_{2}(i i k) q_{1}(i i k)+\psi_{1} \psi_{2}{ }^{s_{2}} q_{1}(i k i) \partial_{1}\left(q_{2}(i i k)\right) q_{1}(i i k) .
\end{aligned}
$$

Now it is easy to check that the $\psi_{1} \psi_{2} \psi_{1^{-}}, \psi_{2} \psi_{1}$-coefficients in the two expressions above are equal to each other using (3.24) and (3.29). For the $\psi_{1} \psi_{2}$-coefficient, we need to use

$$
\partial_{1}\left(q_{2}(i i k)\right)+\partial_{1}\left({ }^{s_{2}} q_{1}(i k i)\right)=\partial_{1}\left(q_{2}(i i k)+{ }^{s_{1}} q_{2}(i i k)\right)=0 .
$$

By a calculation using (3.22) and (2.1), $\partial_{1}\left(p_{2}(i i k)\right)=-{ }^{s_{1}} p_{2}(i i k) p_{2}(i i k)$ and $\partial_{1}\left({ }^{s_{2}} p_{1}(i k i)\right)={ }^{s_{1}} p_{2}(i i k) p_{2}(i i k)$. Also $q_{1}(i i k)=1+y_{2}-y_{1}$ by (3.27) hence $\partial_{1}\left(q_{1}(i i k)\right)=2$. So to check that the $\psi_{1}$-coefficients agree, it suffices to prove

$$
{ }^{s_{1}} p_{2}(i i k) p_{2}(i i k)=p_{2}(i i k)-{ }^{s_{1}} p_{2}(i i k)+{ }^{s_{1}} p_{2}(i i k) p_{2}(i i k)\left(1+y_{2}-y_{1}\right) .
$$

This follows from the power series identity

$$
p_{2}(i i k)-{ }^{s_{1}} p_{2}(i i k)=\left(y_{1}-y_{2}\right)^{s_{1}} p_{2}(i i k) p_{2}(i i k),
$$

which is easily checked from the definition (3.22). For the $\psi_{2}$-coefficients we need to prove

$$
\begin{aligned}
& q_{2}(i i k)\left(p_{2}(i i k)-{ }^{s_{2}} p_{1}(i k i)+{ }^{s_{2}} p_{2}(i k i)^{s_{2}} p_{1}(i k i)\right)= \\
& q_{1}(i i k)\left(\partial_{1}\left(q_{2}(i i k)\right)\left[p_{2}(i i k)-{ }^{s_{2}} p_{1}(i k i)\right]-p_{2}(i i k)^{s_{1}} p_{2}(i i k)^{s_{1}} q_{2}(i i k)\right) .
\end{aligned}
$$

Using (3.46), (3.23) and (3.24), the left hand side of this simplifies to

$$
q_{2}(i i k)\left(y_{1}-y_{2}-1\right)^{s_{1}} p_{2}(i i k) p_{2}(i i k) \text {. }
$$

Using (3.46) again and expanding the $\partial_{1}$, the right hand side equals

$$
\left.q_{1}(i i k)\left(\left({ }^{s_{1}} q_{2}(i i k)-q_{2}(i i k)\right)^{s_{1}} p_{2}(i i k) p_{2}(i i k)-p_{2}(i i k)\right)^{s_{1}} p_{2}(i i k){ }^{s_{1}} q_{2}(i i k)\right) .
$$

Making obvious cancellations and recalling (3.27) this reduces to the left hand side. Finally, for the constant term we want

$$
\begin{aligned}
& -p_{2}(i i k)^{2}-{ }^{s_{1}} p_{2}(i i k) q_{1}(i i k) p_{2}(i i k)^{2}-\left(\psi_{2}^{2}\right)^{s_{2}} q_{2}(i k i)^{s_{2}} p_{1}(i k i) q_{2}(i i k) \\
& \quad=-p_{2}(i i k)-{ }^{s_{1}} p_{2}(i i k) p_{2}(i i k) q_{1}(i i k)+\left(\psi_{1}^{2}\right)^{s_{1}} q_{1}(i i k)^{s_{1}} p_{2}(i i k) q_{1}(i i k) .
\end{aligned}
$$

By (1.14) the third term on the right is zero. By (3.43) the third term on the left equals ${ }^{s_{1}} p_{2}(i i k)\left(1-p_{2}(i i k)^{2}\right)$. Making these substitutions and replacing $q_{1}(i i k)$ by $\left(1+y_{2}-y_{1}\right)$, the desired equality follows from (3.46).

Case 3: $i \neq j=k$. This case is similar to Case 2, so we skip it. 
Case $4: i=k \neq j$. Using the equalities $p_{1}(i i j)=p_{2}(j i i)=1$, and commuting as usual, (3.44) becomes

$$
\begin{aligned}
& -p_{2}(i j i) p_{1}(i j i) p_{2}(i j i)+\psi_{2} q_{2}(i j i) p_{1}(i j i) p_{2}(i j i)+\psi_{1} q_{1}(i j i) p_{2}(i j i) \\
& -\psi_{2} \psi_{1}{ }^{s_{1}} q_{2}(j i i) q_{1}(i j i) p_{2}(i j i)+\psi_{2}{ }^{s_{2}} p_{2}(i i j) q_{2}(i j i)-\left(\psi_{2}^{2}\right)^{s_{2}} q_{2}(i i j) q_{2}(i j i) \\
& -\psi_{1} \psi_{2}{ }^{s_{2} s_{1}} p_{2}(i i j)^{s_{2}} q_{1}(i i j) q_{2}(i j i)-\psi_{2}{ }^{s_{2}} \partial_{1}\left(p_{2}(i i j)\right)^{s_{2}} q_{1}(i i j) q_{2}(i j i) \\
& +\psi_{2} \psi_{1} \psi_{2}{ }^{s_{2} s_{1}} q_{2}(i i j)^{s_{2}} q_{1}(i i j) q_{2}(i j i)+\left(\psi_{2}^{2}\right)^{s_{2}} \partial_{1}\left(q_{2}(i i j)\right)^{s_{2}} q_{1}(i i j) q_{2}(i j i),
\end{aligned}
$$

and (3.45) becomes

$$
\begin{aligned}
& -p_{1}(i j i) p_{2}(i j i) p_{1}(i j i)+\psi_{1} q_{1}(i j i) p_{2}(i j i) p_{1}(i j i)+\psi_{2} q_{2}(i j i) p_{1}(i j i) \\
& -\psi_{1} \psi_{2}{ }^{s_{2}} q_{1}(i i j) q_{2}(i j i) p_{1}(i j i)+\psi_{1}{ }^{s_{1}} p_{1}(j i i) q_{1}(i j i)-\left(\psi_{1}^{2}\right)^{s_{1}} q_{1}(j i i) q_{1}(i j i) \\
& -\psi_{2} \psi_{1}{ }^{s_{1} s_{2}} p_{1}(j i i)^{s_{1}} q_{2}(j i i) q_{1}(i j i)-\psi_{1}{ }^{s_{1}} \partial_{2}\left(p_{1}(j i i)\right)^{s_{1}} q_{2}(j i i) q_{1}(i j i) \\
& +\psi_{1} \psi_{2} \psi_{1}{ }^{s_{1} s_{2}} q_{1}(j i i)^{s_{1}} q_{2}(j i i) q_{1}(i j i)+\left(\psi_{1}^{2}\right)^{s_{1}} \partial_{2}\left(q_{1}(j i i)\right)^{s_{1}} q_{2}(j i i) q_{1}(i j i) .
\end{aligned}
$$

By (1.15), we have that $\psi_{1} \psi_{2} \psi_{1}-\psi_{2} \psi_{1} \psi_{2}=\varepsilon$ where $\varepsilon:=0$ if $i+j, 1$ if $i \rightarrow j,-1$ if $i \leftarrow j$ and $-2 y_{2}+y_{1}+y_{3}$ if $i \rightleftarrows j$. Hence, in view of (3.29), the $\psi_{2} \psi_{1} \psi_{2}$-term cancels with the $\psi_{1} \psi_{2} \psi_{1}$-term, producing the addition of $\varepsilon q_{1}(i j i)^{s_{2}} q_{1}(i i j) q_{2}(i j i)$ to the constant term of the second expression. Now let us compare constant terms. Taking into account (3.43) and (3.29), we need to check that

$$
\begin{array}{r}
-p_{2}(i j i)^{2} p_{1}(i j i)-\left(1-p_{2}(i j i)^{2}\right)+\left(\psi_{2}^{2}\right)^{s_{2}} \partial_{1}\left(q_{2}(i i j)\right)^{s_{2}} q_{1}(i i j) q_{2}(i j i) \\
=-p_{1}(i j i)^{2} p_{2}(i j i)-\left(1-p_{1}(i j i)^{2}\right)+\left(\psi_{1}^{2}\right)^{s_{1}} \partial_{2}\left(q_{1}(j i i)\right)^{s_{1}} q_{2}(j i i) q_{1}(i j i) \\
+\varepsilon q_{1}(i j i)^{s_{2}} q_{1}(i i j) q_{2}(i j i) .
\end{array}
$$

Expanding the $\partial$ 's, we rewrite this as

$$
\begin{aligned}
\left(p_{1}(i j i)-\right. & \left.p_{2}(i j i)\right)\left(p_{1}(i j i) p_{2}(i j i)-p_{1}(i j i)-p_{2}(i j i)\right) \\
& +\left(\psi_{2}^{2}\right)\left({ }^{s_{2} s_{1}} q_{2}(i i j)-{ }^{s_{2}} q_{2}(i i j)\right)^{s_{2}} q_{1}(i i j) q_{2}(i j i) /\left(y_{1}-y_{3}\right) \\
& -\left(\psi_{1}^{2}\right)\left({ }^{s_{1} s_{2}} q_{1}(j i i)-{ }^{s_{1}} q_{1}(j i i)\right)^{s_{1}} q_{2}(j i i) q_{1}(i j i) /\left(y_{1}-y_{3}\right) \\
& -\varepsilon q_{1}(i j i){ }^{s_{2}} q_{1}(i i j) q_{2}(i j i)=0 .
\end{aligned}
$$

Using (3.36) and (3.27), the first term on the left hand side equals

$$
\left(p_{1}(i j i)^{2}-p_{2}(i j i)^{2}\right)^{s_{2}} q_{1}(i j i) /\left(y_{1}-y_{3}\right) .
$$

Using (3.43) and (3.29), the second and third terms equal

$$
\left(q_{1}(i j i) q_{2}(i j i) \psi_{2}^{2}-1+p_{2}(i j i)^{2}\right)^{s_{2}} q_{1}(i i j) /\left(y_{1}-y_{3}\right)
$$

and

$$
-\left(q_{2}(i j i) q_{1}(i j i) \psi_{1}^{2}-1+p_{1}(i j i)^{2}\right)^{s_{2}} q_{1}(j i i) /\left(y_{1}-y_{3}\right),
$$

respectively. Now we observe by (1.14) that

$$
\psi_{2}^{2}-\psi_{1}^{2}=\varepsilon\left(y_{1}-y_{3}\right) .
$$

Making these substitutions, it is then easy to verify the required identity.

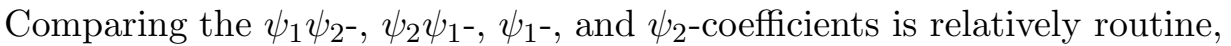
using (3.36) and the fact that ${ }^{s_{1}} \partial_{2}\left(p_{1}(j i i)\right)={ }^{s_{2}} \partial_{1}\left(p_{2}(i i j)\right)=p_{1}(i j i) p_{2}(i j i)$. 
Case 5: $i=j=k$. This case is the most explicit of all since we have the precise formulas for all $p$ 's and $q$ 's. We leave details to the reader.

3.5. Proof of the Main Theorem in the degenerate case. Now we can prove the Main Theorem from the introduction when $q=1$. By Theorem 3.2, there is a homomorphism

$$
\rho: R_{\alpha}^{\Lambda} \rightarrow H_{\alpha}^{\Lambda}
$$

mapping all the formal generators $e(\boldsymbol{i}), y_{r}$ and $\psi_{r}$ to the explicit elements of $H_{\alpha}^{\Lambda}$ with the same names. To prove that $\rho$ is an isomorphism, we construct a two-sided inverse

$$
\sigma: H_{\alpha}^{\Lambda} \rightarrow R_{\alpha}^{\Lambda}
$$

By Theorem 3.3, there is a homomorphism $\sigma: H_{d} \rightarrow R_{\alpha}^{\Lambda}$ mapping the formal generators $x_{r}$ and $s_{r}$ of $H_{d}$ to the elements of $R_{\alpha}^{\Lambda}$ with the same names defined in (3.41) and (3.42). We claim that this homomorphism factors through the natural surjection $H_{d} \rightarrow H_{\alpha}^{\Lambda}$ to give the required inverse homomorphism (3.49). To see this, observe using the orthogonality of the $e(\boldsymbol{i})$ and (1.6) that

$$
\left.\sigma\left(\prod_{i \in I}\left(x_{1}-i\right)^{\left(\Lambda, \alpha_{i}\right)}\right)\right)=\sum_{\boldsymbol{j} \in I^{\alpha}} \prod_{i \in I}\left(y_{1}+j_{1}-i\right)^{\left(\Lambda, \alpha_{i}\right)} e(\boldsymbol{j})=0 .
$$

Hence, $\sigma$ factors through the quotient $H_{d}^{\Lambda}$ of $H_{d}$ from (1.2). To show that $\sigma$ factors further through the surjection $H_{d}^{\Lambda} \rightarrow H_{\alpha}^{\Lambda}$ defined by multiplication by the block idempotent $e_{\alpha}$, we need to show that $\sigma\left(e_{\beta}\right)=0$ for any $\beta \in Q_{+}$ of height $d$ with $\beta \neq \alpha$. Recalling (1.3), this follows immediately from the following lemma.

Lemma 3.4. For any $i \in I^{d}$, we have that

$$
\sigma(e(\boldsymbol{i}))= \begin{cases}e(\boldsymbol{i}) & \text { if } \boldsymbol{i} \in I^{\alpha} \\ 0 & \text { otherwise } .\end{cases}
$$

Proof. To avoid confusion, let us temporarily denote the idempotent $e(\boldsymbol{i}) \in$ $R_{\alpha}^{\Lambda}$ instead by $e(\boldsymbol{i})^{\prime}$. Recall that $e(\boldsymbol{i}) \in H_{d}^{\Lambda}$ is the idempotent characterized by the property that $e(\boldsymbol{i}) M=M_{\boldsymbol{i}}$ for any finite dimensional left $H_{d}^{\Lambda}$-module $M$. The homomorphism $\sigma$ makes $R_{\alpha}^{\Lambda}$ into a finite dimensional left $H_{d}^{\Lambda}$-module. By (3.41), Lemma 2.1 and the relation (1.7), the weight space $\left(R_{\alpha}^{\Lambda}\right)_{i}$ is precisely $e(\boldsymbol{i})^{\prime} R_{\alpha}^{\Lambda}$ if $\boldsymbol{i} \in I^{\alpha}$ and zero otherwise. Hence $\sigma(e(\boldsymbol{i}))$ is an idempotent in $R_{\alpha}^{\Lambda}$ that projects $R_{\alpha}^{\Lambda}$ onto $e(i)^{\prime} R_{\alpha}^{\Lambda}$ if $\boldsymbol{i} \in I^{\alpha}$ and is zero if $\boldsymbol{i} \notin I^{\alpha}$. Hence $\sigma(e(\boldsymbol{i}))=e(\boldsymbol{i})^{\prime}$ if $\boldsymbol{i} \in I^{\alpha}$ and $\sigma(e(\boldsymbol{i}))=0$ otherwise.

This completes the definition of the homomorphism (3.49). To finish the proof of the Main Theorem in the degenerate case it remains to check that $\rho$ and $\sigma$ are two-sided inverses. This follows by the final lemma.

Lemma 3.5. We have that $\sigma \circ \rho=\operatorname{id}_{R_{\alpha}^{\Lambda}}$ and $\rho \circ \sigma=\operatorname{id}_{H_{\alpha}^{\Lambda}}$.

Proof. Since both $\sigma$ and $\rho$ are algebra homomorphisms, it suffices to check that $\sigma \circ \rho$ is the identity on each generator (2.4) of $R_{\alpha}^{\Lambda}$ and that $\rho \circ \sigma$ is 
the identity on each generator (3.1) of $H_{\alpha}^{\Lambda}$. The fact that $\sigma(\rho(e(\boldsymbol{i})))=e(\boldsymbol{i})$ follows from Lemma 3.4. Then using (3.41) and (3.21) we get that

$$
\rho\left(\sigma\left(x_{r}\right)\right)=\sum_{\boldsymbol{i} \in I^{\alpha}} \rho\left(\left(y_{r}+i_{r}\right) e(\boldsymbol{i})\right)=\sum_{\boldsymbol{i} \in I^{\alpha}} x_{r} e(\boldsymbol{i}) .
$$

Since $\sum_{\boldsymbol{i} \in I^{\alpha}} e(\boldsymbol{i})$ is the identity $e_{\alpha} \in H_{\alpha}^{\Lambda}$, this implies $\rho\left(\sigma\left(x_{r}\right)\right)=x_{r}$. The proof that $\rho\left(\sigma\left(s_{r}\right)\right)=s_{r}$ is a similar calculation using (3.42) and (3.32). Finally one checks that $\sigma\left(\rho\left(y_{r}\right)\right)=y_{r}$ and $\sigma\left(\rho\left(\psi_{r}\right)\right)=\psi_{r}$ using the same formulae.

\section{The NON-DEGEnERATE CASE}

4.1. Blocks of cyclotomic Hecke algebras. Now we assume that $q \neq 1$ and let $H_{d}$ be the affine Hecke algebra over $F$ at this parameter. Thus $H_{d}$ has generators

$$
\left\{X_{1}^{ \pm 1}, \ldots, X_{d}^{ \pm 1}\right\} \cup\left\{T_{1}, \ldots, T_{d-1}\right\}
$$

subject to the following relations for all admissible indices:

$$
\begin{aligned}
& X_{r}^{ \pm 1} X_{s}^{ \pm 1}=X_{s}^{ \pm 1} X_{r}^{ \pm 1}, \quad X_{r} X_{r}^{-1}=1 ; \\
& T_{r} X_{r} T_{r}=q X_{r+1}, \quad T_{r} X_{s}=X_{s} T_{r} \quad \text { if } s \neq r, r+1 ; \\
& T_{r}^{2}=(q-1) T_{r}+q ; \\
& T_{r} T_{r+1} T_{r}=T_{r+1} T_{r} T_{r+1}, \quad T_{r} T_{s}=T_{s} T_{r} \quad \text { if }|r-s|>1 .
\end{aligned}
$$

The following relations are easy consequences of the defining relations:

$$
\begin{array}{rlrl}
T_{r}^{-1} & =q^{-1} T_{r}+q^{-1}-1 ; & \\
X_{r} T_{r} & =T_{r} X_{r+1}+(1-q) X_{r+1}, \quad X_{r}^{-1} T_{r}=T_{r} X_{r+1}^{-1}+(q-1) X_{r}^{-1} ; \\
X_{r+1} T_{r} & =T_{r} X_{r}+(q-1) X_{r+1}, \quad X_{r+1}^{-1} T_{r}=T_{r} X_{r}^{-1}+(1-q) X_{r}^{-1} .
\end{array}
$$

We'll use these repeatedly without further note.

Given $\Lambda \in P_{+}$of level $l$ as usual, denote by the same letters $X_{1}^{ \pm 1}, \ldots, X_{d}^{ \pm 1}$ and $T_{1}, \ldots, T_{d-1}$ the images of the generators in the cyclotomic quotient $H_{d}^{\Lambda}$ from (1.2). As goes back to [AK], we have that

$$
\operatorname{dim} H_{d}^{\Lambda}=l^{d} d \text { !, }
$$

just like in the degenerate case. In case $l=1, H_{d}^{\Lambda}$ is the usual finite Hecke algebra associated to the symmetric group $S_{d}$.

Let $M$ be a finite dimensional $H_{d}^{\Lambda}$-module. By [G, Lemma 4.7], the eigenvalues of each $X_{r}$ on $M$ are of the form $q^{i}$ for $i \in I$. So $M$ decomposes as a direct sum $M=\bigoplus_{i \in I^{d}} M_{\boldsymbol{i}}$ of its weight spaces

$$
M_{i}:=\left\{v \in M \mid\left(X_{r}-q^{i_{r}}\right)^{N} v=0 \text { for all } r=1, \ldots, d \text { and } N \gg 0\right\} .
$$

As in (3.7), we have that

$$
T_{r}\left(M_{\boldsymbol{i}}\right) \subseteq M_{\boldsymbol{i}}+M_{s_{r} \cdot \boldsymbol{i}}
$$

Considering the weight space decomposition of the regular module as in 33.1 , we deduce that there is a system $\left\{e(\boldsymbol{i}) \mid \boldsymbol{i} \in I^{d}\right\}$ of mutually orthogonal idempotents in $H_{d}^{\Lambda}$ such that $e(\boldsymbol{i}) M=M_{\boldsymbol{i}}$ for each finite dimensional module $M$. Each $e(\boldsymbol{i})$ lies in the commutative subalgebra generated by $X_{1}^{ \pm 1}, \ldots, X_{d}^{ \pm 1}$, 
all but finitely many of the $e(\boldsymbol{i})$ 's are zero, and their sum is the identity element in $H_{d}^{\Lambda}$.

As goes back to Bernstein, the center $Z\left(H_{d}\right)$ consists of all symmetric polynomials in $X_{1}^{ \pm 1}, \ldots, X_{d}^{ \pm 1}$; see e.g. [G, Proposition 4.1]. So, given also $\alpha \in Q_{+}$of height $d$, the idempotent $e_{\alpha}$ from (1.3) is either zero or it is a central idempotent in $H_{d}^{\Lambda}$. In fact, it follows from [LM] that the nonzero $e_{\alpha}$ 's are precisely the primitive central idempotents of $H_{d}^{\Lambda}$ (although we don't ever use this). So again the algebra $H_{\alpha}^{\Lambda}:=e_{\alpha} H_{d}^{\Lambda}$ from (1.4) is either zero or it is a block of $H_{d}^{\Lambda}$. The commutative subalgebra of $H_{\alpha}^{\Lambda}$ generated by $X_{1}^{ \pm 1}, \ldots, X_{d}^{ \pm 1}$ will be denoted $\operatorname{Pol}_{\alpha}^{\Lambda}$. Each $\operatorname{Pol}_{\alpha}^{\Lambda} e(\boldsymbol{i})$ is an algebra with identity $e(\boldsymbol{i})$. If $X \in \operatorname{Pol}_{\alpha}^{\Lambda}$ is such that $X e(\boldsymbol{i})$ is invertible in $\operatorname{Pol}_{\alpha}^{\Lambda} e(\boldsymbol{i})$, we write $X^{-1} e(\boldsymbol{i})$ for its inverse in $\operatorname{Pol}_{\alpha}^{\Lambda} e(\boldsymbol{i})$. For example, set

$$
X_{r, s}:=X_{r} X_{s}^{-1} \text {. }
$$

Then $\left(1-X_{r, s}\right)^{-1} e(\boldsymbol{i})$ makes sense if $i_{r} \neq i_{s}$.

4.2. Intertwining elements $\boldsymbol{\Phi}_{\boldsymbol{r}}$. For $1 \leq r \leq d$, set

$$
\Phi_{r}:=T_{r}+\sum_{\substack{\boldsymbol{i} \in I^{\alpha} \\ i_{r} \neq i_{r+1}}}(1-q)\left(1-X_{r, r+1}\right)^{-1} e(\boldsymbol{i})+\sum_{\substack{\boldsymbol{i} \in I^{\alpha} \\ i_{r}=i_{r+1}}} e(\boldsymbol{i}) .
$$

This is a slightly modified version of the usual intertwining elements as in [Ro, §2] and [L1, §5.1]:

$$
\Theta_{r}:=T_{r}\left(1-X_{r, r+1}\right)+1-q .
$$

The elements $\Theta_{r}$ have the following nice properties which are checked from the relations; see [L1, Proposition 5.2]:

$$
\begin{aligned}
& \Theta_{r}^{2}=\left(1-q X_{r+1, r}\right)\left(1-q X_{r, r+1}\right) ; \\
& \Theta_{r} X_{r+1}^{ \pm 1}=X_{r}^{ \pm 1} \Theta_{r}, \quad \Theta_{r} X_{s}=X_{s} \Theta_{r} \quad \text { if } s \neq r, r+1 ; \\
& \Theta_{r} \Theta_{r+1} \Theta_{r}=\Theta_{r+1} \Theta_{r} \Theta_{r+1}, \quad \Theta_{r} \Theta_{s}=\Theta_{s} \Theta_{r} \quad \text { if }|r-s|>1 \text {. }
\end{aligned}
$$

The elements $\Phi_{r}$ inherit similar properties:

Lemma 4.1. The intertwining elements satisfy the following relations for all $\boldsymbol{i} \in I^{\alpha}$ and admissible $r, s$ :

$$
\begin{aligned}
& \Phi_{r} e(\boldsymbol{i})=e\left(s_{r} \cdot \boldsymbol{i}\right) \Phi_{r} ; \\
& \Phi_{r} X_{s}=X_{s} \Phi_{r} \quad \text { if } s \neq r, r+1 ; \\
& \Phi_{r} \Phi_{s}=\Phi_{s} \Phi_{r} \quad \text { if }|r-s|>1 ; \\
& \Phi_{r} X_{r+1} e(\boldsymbol{i})= \begin{cases}X_{r} \Phi_{r} e(\boldsymbol{i}) & \text { if } i_{r} \neq i_{r+1}, \\
\left(X_{r} \Phi_{r}+q X_{r+1}-X_{r}\right) e(\boldsymbol{i}) & \text { if } i_{r}=i_{r+1}\end{cases} \\
& X_{r+1} \Phi_{r} e(\boldsymbol{i})= \begin{cases}\Phi_{r} X_{r} e(\boldsymbol{i}) & \text { if } i_{r} \neq i_{r+1}, \\
\left(\Phi_{r} X_{r}+q X_{r+1}-X_{r}\right) e(\boldsymbol{i}) & \text { if } i_{r}=i_{r+1}\end{cases} \\
& \Phi_{r}^{2} e(\boldsymbol{i})= \begin{cases}\frac{\left(X_{r+1}-q X_{r}\right)\left(X_{r}-q X_{r+1}\right)}{\left(X_{r+1}-X_{r}\right)\left(X_{r}-X_{r+1}\right)} e(\boldsymbol{i}) & \text { if } i_{r} \neq i_{r+1}, \\
(1+q) \Phi_{r} e(\boldsymbol{i}) & \text { if } i_{r}=i_{r+1}\end{cases}
\end{aligned}
$$




$$
\Phi_{r} \Phi_{r+1} \Phi_{r}= \begin{cases}\left(\Phi_{r+1} \Phi_{r} \Phi_{r+1}+q \Phi_{r}-q \Phi_{r+1}\right) e(\boldsymbol{i}) & \text { if } i_{r}=i_{r+2}=i_{r+1}, \\ \left(\Phi_{r+1} \Phi_{r} \Phi_{r+1}+Z_{r}\right) e(\boldsymbol{i}) & \text { if } i_{r}=i_{r+2} \neq i_{r+1}, \\ \Phi_{r+1} \Phi_{r} \Phi_{r+1} e(\boldsymbol{i}) & \text { otherwise, }\end{cases}
$$

where $Z_{r}$ denotes $(1-q)^{2} \frac{\left(X_{r} X_{r+2}-X_{r+1}^{2}\right)\left(X_{r} X_{r+1}-q X_{r+1} X_{r+2}\right)}{\left(X_{r}-X_{r+1}\right)^{2}\left(X_{r+1}-X_{r+2}\right)^{2}}$.

Proof. The first equation (4.14) follows by (4.7) and (4.12) using the fact that $\Phi_{r} e(\boldsymbol{i})=\Theta_{r}\left(1-X_{r, r+1}\right)^{-1} e(\boldsymbol{i})$ for $i_{r} \neq i_{r+1}$, in the same way that (3.14) was verified in the proof of Lemma 3.1. The properties (4.15) and (4.16) are clear from definitions. The properties (4.17) and (4.18) come easily from (4.12) and relations in $H_{\alpha}^{\Lambda}$. For (4.19), if $i_{r}=i_{r+1}$, we have $\Phi_{r}^{2} e(\boldsymbol{i})=\left(T_{r}+1\right)^{2} e(\boldsymbol{i})=(1+q) \Phi_{r} e(\boldsymbol{i})$. Now suppose that $i_{r} \neq i_{r+1}$. Then, using (4.11) and (4.12), we have that

$$
\begin{aligned}
\Phi_{r}^{2} e(\boldsymbol{i}) & =\Theta_{r}\left(1-X_{r, r+1}\right)^{-1} \Theta_{r}\left(1-X_{r, r+1}\right)^{-1} e(\boldsymbol{i}) \\
& =\Theta_{r}^{2}\left(1-X_{r+1, r}\right)^{-1}\left(1-X_{r, r+1}\right)^{-1} e(\boldsymbol{i}) \\
& =\left(1-q X_{r+1, r}\right)\left(1-q X_{r, r+1}\right)\left(1-X_{r+1, r}\right)^{-1}\left(1-X_{r, r+1}\right)^{-1} e(\boldsymbol{i}) .
\end{aligned}
$$

Finally, for the proof of (4.20), we assume without loss of generality that $r=1$ and consider five cases like in the proof of Lemma 3.1. Case 4 involves making a lengthy but routine expansion.

\subsection{Khovanov-Lauda generators of $\boldsymbol{H}_{\alpha}^{\boldsymbol{\Lambda}}$ in the non-degenerate case.} Set

$$
y_{r}:=\sum_{i \in I^{\alpha}}\left(1-q^{-i_{r}} X_{r}\right) e(i) .
$$

Note that $y_{1}, \ldots, y_{d} \in \operatorname{Pol}_{\alpha}^{\Lambda}$ are nilpotent, so we are in the situation of (2.3) and can interpret any power series in $F\left[\left[y_{1}, \ldots, y_{d}\right]\right]$ as an element of $\operatorname{Pol}_{\alpha}^{\Lambda}$. It is convenient also to set

$$
y_{r}(\boldsymbol{i}):=q^{i_{r}}\left(1-y_{r}\right) \in F\left[\left[y_{1}, \ldots, y_{d}\right]\right],
$$

so that

$$
X_{r} e(\boldsymbol{i})=y_{r}(\boldsymbol{i}) e(\boldsymbol{i})
$$

for each $i \in I^{\alpha}$. We note further that

$$
\begin{aligned}
y_{r+1}-q y_{r}(\boldsymbol{i}) & =q^{i_{r+1}}\left(y_{r}-y_{r+1}\right) & & \text { if } i_{r} \rightarrow i_{r+1} \text { or } i_{r} \rightleftarrows i_{r+1}, \\
y_{r}(\boldsymbol{i})-q y_{r+1}(\boldsymbol{i}) & =q^{i_{r}}\left(y_{r+1}-y_{r}\right) & & \text { if } i_{r} \leftarrow i_{r+1} \text { or } i_{r} \rightleftarrows i_{r+1}, \\
s_{r} y_{r}\left(s_{r} \cdot \boldsymbol{i}\right) & =y_{r+1}(\boldsymbol{i}) & & \text { for all } \boldsymbol{i} .
\end{aligned}
$$

For any $1 \leq r<d$ and $\boldsymbol{i} \in I^{\alpha}$ we define power series $P_{r}(\boldsymbol{i}) \in F\left[\left[y_{r}, y_{r+1}\right]\right]$ by setting

$$
P_{r}(\boldsymbol{i}):= \begin{cases}1 & \text { if } i_{r}=i_{r+1} \\ (1-q)\left(1-y_{r}(\boldsymbol{i}) y_{r+1}(\boldsymbol{i})^{-1}\right)^{-1} & \text { if } i_{r} \neq i_{r+1}\end{cases}
$$


The following facts are easy to check:

$$
\begin{aligned}
& P_{r}(\boldsymbol{i})+{ }^{s_{r}} P_{r}\left(s_{r} \cdot \boldsymbol{i}\right)=1-q \quad \text { if } i_{r} \neq i_{r+1} ; \\
& { }^{{ }^{s_{r}}} P_{r+1}\left(s_{r} \cdot \boldsymbol{i}\right)={ }^{s_{r+1}} P_{r}\left(s_{r+1} \cdot \boldsymbol{i}\right) \quad \text { for any } \boldsymbol{i} \text {; } \\
& P_{r}(\boldsymbol{i}) e(\boldsymbol{i})= \begin{cases}e(\boldsymbol{i}) & \text { if } i_{r}=i_{r+1} \\
(1-q)\left(1-X_{r, r+1}\right)^{-1} e(\boldsymbol{i}) & \text { if } i_{r} \neq i_{r+1}\end{cases} \\
& \Phi_{r}=\sum_{\boldsymbol{i} \in I^{\alpha}}\left(T_{r}+P_{r}(\boldsymbol{i})\right) e(\boldsymbol{i}) .
\end{aligned}
$$

By explicitly expanding both sides in terms of $y_{1}, \ldots, y_{d}$, one checks that

$$
\left(1-P_{r}(\boldsymbol{i})\right)\left(q+P_{r}(\boldsymbol{i})\right)=\frac{\left(y_{r+1}(\boldsymbol{i})-q y_{r}(\boldsymbol{i})\right)\left(y_{r}(\boldsymbol{i})-q y_{r+1}(\boldsymbol{i})\right)}{\left(y_{r+1}(\boldsymbol{i})-y_{r}(\boldsymbol{i})\right)\left(y_{r}(\boldsymbol{i})-y_{r+1}(\boldsymbol{i})\right)}
$$

for all $\boldsymbol{i} \in I^{\alpha}$ with $i_{r} \neq i_{r+1}$. Note the denominator on the right hand side of (4.32) is a unit in $F\left[\left[y_{r}, y_{r+1}\right]\right]$ so this makes sense.

Fix from now on a choice of invertible elements $Q_{r}(i) \in F\left[\left[y_{r}, y_{r+1}\right]\right]$ with the following properties:

$$
\begin{aligned}
& Q_{r}(\boldsymbol{i})=1-q+q y_{r+1}-y_{r} \quad \text { if } i_{r}=i_{r+1} ; \\
& Q_{r}(\boldsymbol{i})^{s_{r}} Q_{r}\left(s_{r} \cdot \boldsymbol{i}\right)= \begin{cases}\left(1-P_{r}(\boldsymbol{i})\right)\left(q+P_{r}(\boldsymbol{i})\right) & \text { if } i_{r}+i_{r+1}, \\
\frac{\left.\left(1-P_{r}(\boldsymbol{i})\right)\left(q+P_{r}(\boldsymbol{i})\right)\right)}{y_{r}+y_{r}} & \text { if } i_{r} \rightarrow i_{r+1}, \\
\frac{\left(1-P_{r}(\boldsymbol{i})\right)\left(q+P_{r}(\boldsymbol{i})\right)}{y_{r}-y_{r+1}} & \text { if } i_{r} \leftarrow i_{r+1}, \\
\frac{\left(1-P_{r}(\boldsymbol{i})\right)\left(q+P_{r}(\boldsymbol{i})\right)}{\left(y_{r+1}-y_{r}\right)\left(y_{r}-y_{r+1}\right)} & \text { if } i_{r} \rightleftarrows i_{r+1} ;\end{cases} \\
& { }^{s_{r}} Q_{r+1}\left(s_{r+1} s_{r} \cdot \boldsymbol{i}\right)={ }^{s_{r+1}} Q_{r}\left(s_{r} s_{r+1} \cdot \boldsymbol{i}\right) \quad \text { for any } \boldsymbol{i} .
\end{aligned}
$$

In the fractions on the right hand side of (4.34), the fact that the denominators divide the numerators follows because of (4.32) and (4.24)-(4.25). For example, one could choose

$$
Q_{r}(\boldsymbol{i}):= \begin{cases}1-q+q y_{r+1}-y_{r} & \text { if } i_{r}=i_{r+1}, \\ \left.\left(y_{r}(\boldsymbol{i})-q y_{r+1}(\boldsymbol{i})\right)\right) /\left(y_{r}(\boldsymbol{i})-y_{r+1}(\boldsymbol{i})\right) & \text { if } i_{r}+i_{r+1}, \\ \left(y_{r}(\boldsymbol{i})-q y_{r+1}(\boldsymbol{i})\right) /\left(y_{r}(\boldsymbol{i})-y_{r+1}(\boldsymbol{i})\right)^{2} & \text { if } i_{r} \rightarrow i_{r+1}, \\ q^{i_{r}} & \text { if } i_{r} \leftarrow i_{r+1}, \\ q^{i_{r}} /\left(y_{r}(\boldsymbol{i})-y_{r+1}(\boldsymbol{i})\right) & \text { if } i_{r} \rightleftarrows i_{r+1},\end{cases}
$$

which satisfy (4.34) by another application of (4.32) and (4.24)-(4.26).

Now, the Khovanov-Lauda generators in the non-degenerate case are

$$
\left\{e(\boldsymbol{i}) \mid \boldsymbol{i} \in I^{\alpha}\right\} \cup\left\{y_{1}, \ldots, y_{d}\right\} \cup\left\{\psi_{1}, \ldots, \psi_{d-1}\right\},
$$

where $y_{r}$ is the element defined by (4.21) and

$$
\psi_{r}:=\sum_{\boldsymbol{i} \in I^{\alpha}} \Phi_{r} Q_{r}(\boldsymbol{i})^{-1} e(\boldsymbol{i})=\sum_{\boldsymbol{i} \in I^{\alpha}}\left(T_{r}+P_{r}(\boldsymbol{i})\right) Q_{r}(\boldsymbol{i})^{-1} e(\boldsymbol{i}) .
$$

Theorem 4.2. The elements (4.37) of $H_{\alpha}^{\Lambda}$ satisfy the defining relations (1.6)-(1.15) of the cyclotomic Khovanov-Lauda algebra.

Proof. The checks of (1.6)-(1.11) go in the same way as in the proof of Theorem 3.2, these are easy so we omit them. For (1.13), we have that

$$
y_{r+1} \psi_{r} e(\boldsymbol{i})=\left(1-q^{-i_{r}} X_{r+1}\right) \Phi_{r} Q_{r}(\boldsymbol{i})^{-1} e(\boldsymbol{i}) .
$$


If $i_{r} \neq i_{r+1}$, this equals $\Phi_{r} Q_{r}(\boldsymbol{i})^{-1}\left(1-q^{-i_{r}} X_{r}\right) e(\boldsymbol{i})=\psi_{r} y_{r} e(\boldsymbol{i})$ by (4.18). If $i_{r}=i_{r+1}$, then (4.39) gives

$$
\begin{aligned}
y_{r+1} \psi_{r} e(\boldsymbol{i}) & =\left(1-q^{-i_{r}} X_{r+1}\right)\left(T_{r}+1\right) Q_{r}(\boldsymbol{i})^{-1} e(\boldsymbol{i}) \\
& =\left(\left(T_{r}+1\right)\left(1-q^{-i_{r}} X_{r}\right)+q^{-i_{r}} X_{r}-q^{1-i_{r}} X_{r+1}\right) Q_{r}(\boldsymbol{i})^{-1} e(\boldsymbol{i}) \\
& =\left(\psi_{r} y_{r}+1\right) e(\boldsymbol{i}),
\end{aligned}
$$

since $\left(q^{-i_{r}} X_{r}-q^{1-i_{r}} X_{r+1}\right) e(\boldsymbol{i})=Q_{r}(\boldsymbol{i}) e(\boldsymbol{i})$. The proof of (1.12) is similar.

As we have now verified (1.10), (1.12) and (1.13), we can make use of the identity (2.6) in $H_{\alpha}^{\Lambda}$ when necessary. For (1.14), using (1.8), we have:

$$
\psi_{r}^{2} e(\boldsymbol{i})=\Phi_{r} Q_{r}\left(s_{r} \cdot \boldsymbol{i}\right)^{-1} \psi_{r} e(\boldsymbol{i}) .
$$

If $i_{r} \neq i_{r+1}$, then by (2.6), this becomes

$$
\Phi_{r} \psi_{r}^{s_{r}} Q_{r}\left(s_{r} \cdot \boldsymbol{i}\right)^{-1} e(\boldsymbol{i})=\Phi_{r}^{2}\left(Q_{r}(\boldsymbol{i})^{s_{r}} Q_{r}\left(s_{r} \cdot \boldsymbol{i}\right)\right)^{-1} e(\boldsymbol{i}) .
$$

By (4.19), (4.23) and (4.32), we have that $\Phi_{r}^{2} e(\boldsymbol{i})=\left(1-P_{r}(\boldsymbol{i})\right)\left(q+P_{r}(\boldsymbol{i})\right) e(\boldsymbol{i})$, hence this expression simplifies to give the right hand side of (1.14) by (4.34). Now, let $i_{r}=i_{r+1}$. Then, using (2.6) again, (4.40) becomes

$$
\begin{aligned}
\psi_{r}^{2} e(\boldsymbol{i}) & =\left(T_{r}+1\right)\left(1-q+q y_{r+1}-y_{r}\right)^{-1} \psi_{r} e(\boldsymbol{i}) \\
& =\left(T_{r}+1\right)\left(T_{r}-q\right)\left(1-q+q y_{r}-y_{r+1}\right)^{-1}\left(1-q+q y_{r+1}-y_{r}\right)^{-1} e(\boldsymbol{i}),
\end{aligned}
$$

which is zero by (4.4).

Finally we prove (1.15). Let us also stop writing $e(\boldsymbol{i})$ on the right of all formulas. Assume without loss of generality that $r=1, d=3$, denote $i:=i_{1}, j:=i_{2}, k:=i_{3}$, and consider the usual five cases.

Case 1: $i, j, k$ all distinct. This is exactly the same calculation as Case 1 from the proof of Theorem 3.2, one needs to use (2.6), (4.20) and (4.35).

Case 2: $i=j \neq k$. This is exactly the same calculation as Case 2 from the proof of Theorem 3.2 .

Case 3: $i \neq j=k$. Similar.

Case 4: $i=k \neq j$. Expanding as in Case 4 from the proof of Theorem 3.2 and using (4.19), (4.20), (4.33) and (4.35), we get that $\psi_{1} \psi_{2} \psi_{1}-\psi_{2} \psi_{1} \psi_{2}=$ $A+B-C$ where

$$
\begin{aligned}
A & =(1-q)^{2} \frac{\left(X_{1} X_{3}-X_{2}^{2}\right)\left(X_{1} X_{2}-q X_{2} X_{3}\right)}{\left(X_{1}-X_{2}\right)^{2}\left(X_{2}-X_{3}\right)^{2}}\left(Q_{1}(i j i)^{s_{1}} Q_{2}(j i i) Q_{2}(i j i)\right)^{-1}, \\
B & =\frac{\left(X_{2}-q X_{1}\right)\left(X_{1}-q X_{2}\right)}{\left(X_{2}-X_{1}\right)\left(X_{1}-X_{2}\right)} Q_{1}(i j i)^{-1}\left({ }^{s_{1}} \partial_{2}\left(Q_{1}(j i i)^{-1}\right)\right), \\
C & =\frac{\left(X_{3}-q X_{2}\right)\left(X_{2}-q X_{3}\right)}{\left(X_{3}-X_{2}\right)\left(X_{2}-X_{3}\right)} Q_{2}(i j i)^{-1}\left({ }^{s_{2}} \partial_{1}\left(Q_{2}(i i j)^{-1}\right)\right) .
\end{aligned}
$$

Noting that ${ }^{s_{1}} Q_{2}(j i i)=q^{-i}\left(X_{1}-q X_{3}\right)$, we get that

$$
A=q^{i}(1-q)^{2} \frac{\left(X_{1} X_{3}-X_{2}^{2}\right) X_{2}}{\left(X_{1}-X_{2}\right)^{2}\left(X_{2}-X_{3}\right)^{2}} Q_{1}(i j i)^{-1} Q_{2}(i j i)^{-1} \text {. }
$$


Expanding the $\partial$ 's and using (4.35), we also have that

$$
\begin{aligned}
& B=\frac{\left(X_{2}-q X_{1}\right)\left(X_{1}-q X_{2}\right)}{\left(X_{2}-X_{1}\right)\left(X_{1}-X_{2}\right)} \cdot \frac{Q_{1}(i j i)^{-1} Q_{2}(i j i)^{-1}-\left(Q_{1}(i j i)^{s_{1}} Q_{1}(j i i)\right)^{-1}}{y_{1}-y_{3}}, \\
& C=\frac{\left(X_{3}-q X_{2}\right)\left(X_{2}-q X_{3}\right)}{\left(X_{3}-X_{2}\right)\left(X_{2}-X_{3}\right)} \cdot \frac{Q_{1}(i j i)^{-1} Q_{2}(i j i)^{-1}-\left(Q_{2}(i j i)^{s_{2}} Q_{2}(i i j)\right)^{-1}}{y_{1}-y_{3}} .
\end{aligned}
$$

Note also that $y_{1}-y_{3}=-q^{-i}\left(X_{1}-X_{3}\right)$, and by a direct expansion we have the identity

$$
\begin{aligned}
(1-q)^{2} \frac{\left(X_{1} X_{3}-X_{2}^{2}\right) X_{2}}{\left(X_{1}-X_{2}\right)^{2}\left(X_{2}-X_{3}\right)^{2}}+\frac{\left(X_{2}-q X_{1}\right)\left(X_{1}-q X_{2}\right)}{\left(X_{1}-X_{2}\right)^{2}\left(X_{1}-X_{3}\right)} & \\
-\frac{\left(X_{3}-q X_{2}\right)\left(X_{2}-q X_{3}\right)}{\left(X_{2}-X_{3}\right)^{2}\left(X_{1}-X_{3}\right)} & =0 .
\end{aligned}
$$

Combining these things gives that

$$
\begin{aligned}
A+B-C=-\frac{\left(X_{2}-q X_{1}\right)\left(X_{1}-q X_{2}\right)}{\left(X_{2}-X_{1}\right)\left(X_{1}-X_{2}\right)} \cdot \frac{\left(Q_{1}(i j i)^{s_{1}} Q_{1}\left(s_{1} \cdot i j i\right)\right)^{-1}}{y_{1}-y_{3}} \\
+\frac{\left(X_{3}-q X_{2}\right)\left(X_{2}-q X_{3}\right)}{\left(X_{3}-X_{2}\right)\left(X_{2}-X_{3}\right)} \cdot \frac{\left(Q_{2}(i j i)^{s_{2}} Q_{2}\left(s_{2} \cdot i j i\right)\right)^{-1}}{y_{1}-y_{3}} .
\end{aligned}
$$

Now use (4.23), (4.32) and (4.34) to deduce that this equals 0 if $i+j, 1$ if $i \rightarrow j,-1$ if $i \leftarrow j$ or $-2 y_{2}+y_{1}+y_{3}$ if $i \rightleftarrows j$. This imples (1.15).

Case 5: $i=j=k$. Exercise.

4.4. Hecke generators of $\boldsymbol{R}_{\boldsymbol{\alpha}}^{\boldsymbol{\Lambda}}$. Once again, we let $R_{\alpha}^{\Lambda}$ be the cyclotomic Khovanov-Lauda algebra from $\$ 2.2$, Using the homomorphism (2.5), we can regard the power series $P_{r}(\boldsymbol{i}), Q_{r}(\boldsymbol{i})$ and $y_{r}(\boldsymbol{i})$ from $\$ 4.3$ as elements of $R_{\alpha}^{\Lambda}$. The Hecke generators of $R_{\alpha}^{\Lambda}$ are the elements

$$
\left\{X_{1}, \ldots, X_{d}\right\} \cup\left\{T_{1}, \ldots, T_{d-1}\right\}
$$

where

$$
\begin{aligned}
X_{r} & :=\sum_{\boldsymbol{i} \in I^{\alpha}} y_{r}(\boldsymbol{i}) e(\boldsymbol{i})=\sum_{\boldsymbol{i} \in I^{\alpha}} q^{i_{r}}\left(1-y_{r}\right) e(\boldsymbol{i}), \\
T_{r} & :=\sum_{\boldsymbol{i} \in I^{\alpha}}\left(\psi_{r} Q_{r}(\boldsymbol{i})-P_{r}(\boldsymbol{i})\right) e(\boldsymbol{i}) .
\end{aligned}
$$

We often need the following consequence of (1.14) and (4.34):

$$
\psi_{r}^{2} Q_{r}(\boldsymbol{i})^{s_{r}} Q_{r}\left(s_{r} \cdot \boldsymbol{i}\right) e(\boldsymbol{i})=\left(1-P_{r}(\boldsymbol{i})\right)\left(q+P_{r}(\boldsymbol{i})\right) e(\boldsymbol{i}) .
$$

Now we are ready to make the final set of computations.

Theorem 4.3. The elements (4.41) of $R_{\alpha}^{\Lambda}$ satisfy the defining relations (4.2) - 4.5) of the affine Hecke algebra $H_{d}$.

Proof. The relations (4.2), (4.3) for $s \neq r, r+1$, and (4.5) for $|r-s|>1$ are obvious. Next we check (4.4), i.e. $T_{r}^{2} e(\boldsymbol{i})=(q-1) T_{r} e(\boldsymbol{i})+q e(\boldsymbol{i})$ for all 
$\boldsymbol{i} \in I^{\alpha}$. By exactly the same calculation as in the proof of Theorem 3.3 , we have that

$$
T_{r}^{2}=\left(\psi_{r} Q_{r}\left(s_{r} \cdot \boldsymbol{i}\right) \psi_{r} Q_{r}(\boldsymbol{i})-P_{r}\left(s_{r} \cdot \boldsymbol{i}\right) \psi_{r} Q_{r}(\boldsymbol{i})-\psi_{r} Q_{r}(\boldsymbol{i}) P_{r}(\boldsymbol{i})+P_{r}(\boldsymbol{i})^{2}\right) e(\boldsymbol{i}) .
$$

If $i_{r}=i_{r+1}$ we use the facts $\psi_{r}^{2} e(\boldsymbol{i})=0$ and $\partial_{r}\left(1-q+q y_{r+1}-y_{r}\right)=q+1$ combined with (2.6) to deduce that

$$
\begin{aligned}
T_{r}^{2} e(\boldsymbol{i}) & =\left(\psi_{r}\left(1-q+q y_{r+1}-y_{r}\right) \psi_{r} Q_{r}(\boldsymbol{i})-2 \psi_{r} Q_{r}(\boldsymbol{i})+1\right) e(\boldsymbol{i}) \\
& =\left((q+1) \psi_{r} Q_{r}(\boldsymbol{i})-2 \psi_{r} Q_{r}(\boldsymbol{i})+1\right) e(\boldsymbol{i}) \\
& =\left((q-1)\left(\psi_{r} Q_{r}(\boldsymbol{i})-1\right)+q\right) e(\boldsymbol{i})=\left((q-1) T_{r}+q\right) e(\boldsymbol{i}) .
\end{aligned}
$$

If $i=j$ then by (2.6), (4.44) and (4.28) we get instead that

$$
\begin{aligned}
T_{r}^{2} & =\left(\left(\psi_{r}^{2}\right)^{s_{r}} Q_{r}\left(s_{r} \cdot \boldsymbol{i}\right) Q_{r}(\boldsymbol{i})-\psi_{r}\left(P_{r}(\boldsymbol{i})+{ }^{s_{r}} P_{r}\left(s_{r} \cdot \boldsymbol{i}\right)\right) Q_{r}(\boldsymbol{i})+P_{r}(\boldsymbol{i})^{2}\right) e(\boldsymbol{i}) \\
& =\left(\left(1-P_{r}(\boldsymbol{i})\right)\left(q+P_{r}(\boldsymbol{i})\right)+(q-1) \psi_{r} Q_{r}(\boldsymbol{i})+P_{r}(\boldsymbol{i})^{2}\right) e(\boldsymbol{i}) \\
& =\left((q-1)\left(\psi_{r} Q_{r}(\boldsymbol{i})-P_{r}(\boldsymbol{i})\right)+q\right) e(\boldsymbol{i})=\left((q-1) T_{r}+q\right) e(\boldsymbol{i}) .
\end{aligned}
$$

This completes the proof of the quadratic relation.

Next consider the remaining mixed relation from (4.3). As we have already checked the quadratic relation, it suffices to show that $X_{r} T_{r} e(\boldsymbol{i})=\left(T_{r}+1-\right.$ q) $X_{r+1} e(\boldsymbol{i})$ for each $\boldsymbol{i}$. Using (2.6), (4.26) and the fact that $\partial_{r}\left(y_{r}(\boldsymbol{i})\right)=q^{i_{r}}$, we get that

$$
\begin{aligned}
X_{r} T_{r} e(\boldsymbol{i}) & =\left(y_{r}\left(s_{r} \cdot \boldsymbol{i}\right) \psi_{r} Q_{r}(\boldsymbol{i})-y_{r}(\boldsymbol{i}) P_{r}(\boldsymbol{i})\right) e(\boldsymbol{i}) \\
& =\left(\psi_{r} y_{r+1}(\boldsymbol{i}) Q_{r}(\boldsymbol{i})+\delta_{i_{r}, i_{r+1}} q^{i_{r}} Q_{r}(\boldsymbol{i})-y_{r}(\boldsymbol{i}) P_{r}(\boldsymbol{i})\right) e(\boldsymbol{i}), \\
\left(T_{r}+1-q\right) X_{r+1} e(\boldsymbol{i}) & =\left(\psi_{r} Q_{r}(\boldsymbol{i})-P_{r}(\boldsymbol{i})+1-q\right) y_{r+1}(\boldsymbol{i}) e(\boldsymbol{i}) .
\end{aligned}
$$

Considering the two cases $i_{r} \neq i_{r+1}$ and $i_{r}=i_{r+1}$ separately, it's now an easy exercise to check these two expressions are equal using (4.22), (4.27) and (4.33).

This just leaves the braid relations. We assume that $r=1, d=3$, set $i:=i_{1}, j:=j_{1}, k:=k_{1}$, and need to show that $T_{2} T_{1} T_{2} e(i j k)=T_{1} T_{2} T_{1} e(i j k)$. As usual we stop writing $e(i j k)$ on the right of all expressions. To start with, the left (resp. right) hand side of the identity to be checked expands to exactly the same expression as (3.44) (resp. (3.45)), with all $p_{r}$ and $q_{r}$ there replaced by $P_{r}$ and $Q_{r}$. Then we consider the usual five cases.

Case 1: $i, j, k$ are all different. This is entirely similar to Case 1 in the proof of Theorem 3.3. When equating $\psi_{1^{-}}, \psi_{2^{-}}$and constant coefficients at the end, everything reduces to the following three identities:

$$
\begin{aligned}
{ }^{s_{1}} P_{2}(j i k) P_{2}(i j k) & =P_{1}(i j k) P_{2}(i j k)+{ }^{s_{1}} P_{1}(j i k)^{s_{1}} P_{2}(j i k), \\
{ }^{s_{2}} P_{1}(i k j) P_{1}(i j k) & =P_{1}(i j k) P_{2}(i j k)+{ }^{s_{2}} P_{1}(i k j)^{s_{2}} P_{2}(i k j), \\
P_{1}(i j k) P_{2}(i j k)^{2}+ & \left(\psi_{2}^{2}\right)^{s_{2}} Q_{2}(i k j)^{s_{2}} P_{1}(i k j) Q_{2}(i j k) \\
& =P_{1}(i j k)^{2} P_{2}(i j k)+\left(\psi_{1}^{2}\right)^{s_{1}} Q_{1}(j i k)^{s_{1}} P_{2}(j i k) Q_{1}(i j k) .
\end{aligned}
$$

The first two of these are easily checked from (4.27) and (4.26). The last one follows using (4.44) too. 
Case 2: $i=j \neq k$. Again, the initial expansions made in Case 2 of Theorem 3.3 are valid in the present situation. We need to equate coefficients on both sides of these expansions. As before, this is easy until we get to

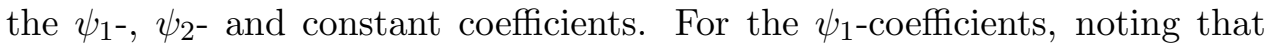
$\partial_{1}\left(Q_{1}(i i k)\right)=1+q$, we have to show that

$$
\partial_{1}\left(P_{2}(i i k)\right) Q_{1}(i i k)=P_{2}(i i k)-q^{s_{1}} P_{2}(i i k)-P_{2}(i i k)^{s_{1}} P_{2}(i i k) .
$$

This can be checked by brute force, expanding both sides fully using (4.27), (4.22) and (4.33). Next, for the $\psi_{2}$-coefficients, we need to show that

$$
\begin{aligned}
& \left(P_{2}(i i k)-{ }^{s_{2}} P_{1}(i k i)+{ }^{s_{2}} P_{1}(i k i){ }^{s_{2}} P_{2}(i k i)\right) Q_{2}(i i k)= \\
& \quad\left[\partial_{1}\left(Q_{2}(i i k)\right)\left(P_{2}(i i k)-{ }^{s_{2}} P_{1}(i k i)\right)-{ }^{s_{1}} Q_{2}(i i k) \partial_{1}\left({ }^{s_{2}} P_{1}(i k i)\right)\right] Q_{1}(i i k) .
\end{aligned}
$$

Replacing ${ }^{s_{2}} P_{1}(i k i)$ with ${ }^{s_{1}} P_{2}(i i k)$ everywhere and using (4.28) to rewrite the term ${ }^{s_{2}} P_{2}(i k i)$ as $1-q-P_{2}(i i k)$, this identity is equivalent to

$$
\begin{aligned}
& \left.\left(P_{2}(i i k)-q^{s_{1}} P_{2}(i i k)-P_{2}(i i k)\right)^{s_{1}} P_{2}(i i k)\right) Q_{2}(i i k)= \\
& \quad\left[\partial_{1}\left(Q_{2}(i i k)\right)\left(P_{2}(i i k)-{ }^{s_{1}} P_{2}(i i k)\right)-{ }^{s_{1}} Q_{2}(i i k) \partial_{1}\left({ }^{s_{1}} P_{2}(i i k)\right)\right] Q_{1}(i i k) .
\end{aligned}
$$

Now we expand the $\partial_{1}$ 's on the right hand side of (4.46) to see that it equals

$$
\frac{{ }^{s_{1}} P_{2}(i i k)-P_{2}(i i k)}{y_{1}-y_{2}} Q_{1}(i i k) Q_{2}(i i k) \text {. }
$$

This equals $\partial_{1}\left(P_{2}(i i k)\right) Q_{1}(i i k) Q_{2}(i i k)$ which by (4.45) is equal to the left hand side of (4.46) . Finally, to check the constant coefficients, we need to show that

$$
\begin{aligned}
P_{2}(i i k)\left(1-P_{2}(i i k)\right)-\left(1-P_{2}(i i k)\right)(q & \left.+P_{2}(i i k)\right)^{s_{2}} P_{1}(i k i) \\
& =\partial_{1}\left(P_{2}(i i k)\right) Q_{1}(i i k)\left(1-P_{2}(i i k)\right),
\end{aligned}
$$

where we have used (4.44) and the observation that $\psi_{1}^{2}=0$ by (1.14). Noting that ${ }^{s_{2}} P_{1}(i k i)={ }^{s_{1}} P_{2}(i i k)$, this follows from (4.45) on multiplying both sides by $\left(1-P_{2}(i i k)\right)$.

Case 3: $i \neq j=k$. Similar.

Case 4: $i=k \neq j$. Again we follow the calculation from Case 4 of Theorem 3.3. Let $\varepsilon:=0$ if $i+j, 1$ if $i \rightarrow j,-1$ if $i \leftarrow j$ and $-2 y_{2}+y_{1}+y_{3}$ if $i \rightleftarrows j$. In view of (3.29), the $\psi_{2} \psi_{1} \psi_{2}$-term cancels with the $\psi_{1} \psi_{2} \psi_{1}$-term, producing the addition of $\varepsilon q_{1}(i j i)^{s_{2}} q_{1}(i i j) q_{2}(i j i)$ to the constant term of the second expression. Now we just consider the constant coefficients, all the other coefficients being routine. Using (4.44), we need to show that

$$
\begin{aligned}
-P_{2}(i j i)^{2} P_{1}(i j i)-\left(1-P_{2}(i j i)\right)( & \left.+P_{2}(i j i)\right) \\
& +\left(\psi_{2}^{2}\right)^{s_{2}} \partial_{1}\left(Q_{2}(i i j)\right)^{s_{2}} Q_{1}(i i j) Q_{2}(i j i)
\end{aligned}
$$

equals

$$
\begin{aligned}
& -P_{1}(i j i)^{2} P_{2}(i j i)-\left(1-P_{1}(i j i)\right)\left(q+P_{1}(i j i)\right)+ \\
& \left(\psi_{1}^{2}\right)^{s_{1}} \partial_{2}\left(Q_{1}(j i i)\right)^{s_{1}} Q_{2}(j i i) Q_{1}(i j i)+\varepsilon Q_{1}(i j i)^{s_{2}} Q_{1}(i i j) Q_{2}(i j i) .
\end{aligned}
$$


Expanding the $\partial$ 's, this means we have to show that

$$
\begin{aligned}
& P_{1}(i j i) P_{2}(i j i)\left(P_{1}(i j i)-P_{2}(i j i)\right) \\
& +\left(1-P_{1}(i j i)\right)\left(q+P_{1}(i j i)\right)-\left(1-P_{2}(i j i)\right)\left(q+P_{2}(i j i)\right) \\
& \quad+\left(\psi_{2}^{2}\right) \frac{Q_{1}(i j i) Q_{2}(i j i)-{ }^{s_{2}} Q_{2}(i i j) Q_{2}(i j i)}{y_{1}-y_{3}} s_{2} Q_{1}(i i j) \\
& \quad-\left(\psi_{1}^{2}\right) \frac{Q_{1}(i j i) Q_{2}(i j i)-Q_{1}(i j i)^{s_{1}} Q_{1}(j i i)}{y_{1}-y_{3}} s_{2} Q_{1}(i i j) \\
& \quad-\varepsilon Q_{1}(i j i) Q_{2}(i j i)^{s_{2}} Q_{1}(i i j)=0 .
\end{aligned}
$$

Now we use (3.47) and (4.44) and simplify to get that the left hand side of this expression equals

$$
\begin{array}{r}
\left(\left(1-P_{1}(i j i)\right)\left(q+P_{1}(i j i)\right)-\left(1-P_{2}(i j i)\right)\left(q+P_{2}(i j i)\right) \frac{s_{2} Q_{1}(i i j)+y_{1}-y_{3}}{y_{1}-y_{3}}\right. \\
+P_{1}(i j i) P_{2}(i j i)\left(P_{1}(i j i)-P_{2}(i j i)\right) .
\end{array}
$$

Simplifying the first term using (4.33) then cancelling $\left(P_{1}(i j i)-P_{2}(i j i)\right)$ everywhere, this reduces to checking

$$
(1-q)\left(1-q-P_{1}(i j i)-P_{2}(i j i)\right)\left(1-y_{3}\right)+P_{1}(i j i) P_{2}(i j i)\left(y_{1}-y_{3}\right)=0,
$$

which is straightforward using (4.27) then (4.22).

Case 5: $i=j=k$. Exercise.

4.5. Proof of the Main Theorem in the non-degenerate case. Finally we can prove the Main Theorem from the introduction for $q \neq 1$. By Theorem 4.2, there is a homomorphism

$$
\rho: R_{\alpha}^{\Lambda} \rightarrow H_{\alpha}^{\Lambda}
$$

mapping all the formal generators $e(\boldsymbol{i}), y_{r}$ and $\psi_{r}$ to the explicit elements of $H_{\alpha}^{\Lambda}$ with the same names. This homomorphism is an isomorphism because it has a two-sided inverse

$$
\sigma: H_{\alpha}^{\Lambda} \rightarrow R_{\alpha}^{\Lambda}
$$

mapping the generators $T_{r}$ and $X_{r}$ to the explicit elements of $R_{\alpha}^{\Lambda}$ with the same names. This statement follows by Theorem 4.3 and arguments that are entirely similar to those in 3.5 .

\section{EXAmPle: Young'S SEMI-NORMAl FORM}

In this section we make a rather drastic special assumption: $\Lambda=\Lambda_{0}$ and $e=0$. With this assumption, the degenerate cyclotomic Hecke algebra $H_{d}^{\Lambda}$ is equal to the group algebra $F S_{d}$ of the symmetric group if $q=1$ or the corresponding Iwahori-Hecke algebra if $q \neq 1$. Either way, $H_{d}^{\Lambda}$ is a semisimple algebra, so its blocks are matrix algebras. We want to explain how in this special case our results basically reduce to the classical Young's semi-normal form. From this point of view, one can think loosely of our Main Theorem as a replacement for Young's semi-normal form when the blocks are not simple. 
Let $\lambda$ be a partition of $d$. We identify $\lambda$ with its Young diagram drawn in the usual English notation. The residue of the box of $\lambda$ in row $i$ and column $j$ is defined to be $j-i \in I$. By a $\lambda$-tableau we mean a diagram obtained by filling the boxes of $\lambda$ with the entries $1, \ldots, d$ (each appearing exactly once). Let $\mathscr{T}(\lambda)$ denote the usual set of all standard $\lambda$-tableaux, i.e. the $\lambda$-tableaux whose entries are strictly increasing both along rows from left to right and down columns from top to bottom. The symmetric group $S_{d}$ acts naturally on the set of all $\lambda$-tableaux by its action on the entries, but it does not preserve the subset $\mathscr{T}(\lambda)$ of standard $\lambda$-tableaux.

To any $\mathrm{T} \in \mathscr{T}(\lambda)$ we associate its residue sequence $\boldsymbol{i}^{\mathrm{T}}:=\left(i_{1}, \ldots, i_{d}\right)$, where $i_{m}$ is the residue of the box of $\mathrm{T}$ containing the entry $m$. We define the weight of a partition $\lambda$ to be the weight $\alpha_{i_{1}}+\cdots+\alpha_{i_{d}} \in Q_{+}$where $\left(i_{1}, \ldots, i_{d}\right)$ is the residue sequence of any standard $\lambda$-tableau. The partition $\lambda$ can be uniquely recovered from its weight.

Now fix a partition $\lambda$ of $d$ of weight $\alpha$. We use Khovanov-Lauda generators to construct a module $S(\lambda)$ over the block $H_{\alpha}^{\Lambda}$ of the symmetric group $F S_{d}$. As a vector space, we let

$$
S(\lambda):=\bigoplus_{\mathrm{T} \in \mathscr{T}(\lambda)} F v_{\mathrm{T}} .
$$

The action of the Khovanov-Lauda generators on this basis is defined as follows:

$$
\begin{aligned}
e(\boldsymbol{i}) v_{\mathrm{T}} & := \begin{cases}v_{\mathrm{T}} & \text { if } \boldsymbol{i}^{\mathrm{T}}=\boldsymbol{i}, \\
0 & \text { otherwise }\end{cases} \\
y_{r} v_{\mathrm{T}} & :=0 ; \\
\psi_{r} v_{\mathrm{T}} & := \begin{cases}v_{s_{r} \mathrm{~T}} & \text { if } s_{r} \mathrm{~T} \in \mathscr{T}(\lambda), \\
0 & \text { otherwise. }\end{cases}
\end{aligned}
$$

It is now very easy to check that the relations (1.6)-(1.15) are satisfied, hence applying our Main Theorem in this very special case we get an $H_{\alpha}^{\Lambda}$-action on $S(\lambda)$. Moreover, if $\mathrm{S}, \mathrm{T} \in \mathscr{T}(\lambda)$ then one can obtain $\mathrm{T}$ from $\mathrm{S}$ by a series of basic transpositions so that on each step we still have a standard tableau; see for example [K1, Lemma 2.2.8]. It follows that the $H_{\alpha}^{\Lambda}$-module $S(\lambda)$ is irreducible.

Since $H_{\alpha}^{\Lambda}$ is a simple matrix algebra, our construction yields an isomorphism $H_{\alpha}^{\Lambda} \cong \operatorname{End}_{F}(S(\lambda))$. Letting $\lambda$ vary over all partitions of $d$, we get a full set of pairwise inequivalent irreducible $H_{\alpha}^{\Lambda}$-modules in this way. Finally, if $q=1$ and we use (3.42), we obtain formulas for the action of the standard generators $s_{1}, \ldots, s_{d-1}$ on each $S(\lambda)$ which are exactly Young's formulas. On the other hand if $q \neq 1$ and we use (4.43) we obtain formulas for the action of $T_{1}, \ldots, T_{d-1}$ on $S(\lambda)$ which are essentially the formulae going back to Hoefsmit $[\mathrm{H}]$; see also [Ra and [W].

Replacing the set $\mathscr{T}(\lambda)$ with the set $\mathscr{T}_{e}(\lambda)$ of e-standard tableaux, the construction of the irreducible module $S(\lambda)$ by the formulae (5.2) - (5.4) can be generalized to include the so-called completely splittable irreducible representations of the symmetric group in characteristic $e>0$ (and their $q$-analogues at roots of unity); see [K2] and $[\mathrm{Ru}$. 


\section{BASE CHANGE}

Finally we explain an application of our main result to base change. Fix $\Lambda \in P_{+}$of level $l$ and $\alpha \in Q_{+}$of height $d$. Henceforth we will denote the algebra $H_{\alpha}^{\Lambda}\left(\right.$ resp. $\left.H_{d}^{\Lambda}\right)$ instead by $H_{\alpha}^{\Lambda}(F)$ (resp. $\left.H_{d}^{\Lambda}(F)\right)$, as we are going to allow the ground field to change. Also make a choice of Khovanov-Lauda generators for $H_{\alpha}^{\Lambda}(F)$ according to (3.31) if $q=1$ or (4.37) if $q \neq 1$. To start with we explain how to descend from the field $F$ to its prime subfield $E$.

Theorem 6.1. Let $E$ be the prime subfield of $F$. Let $H_{\alpha}^{\Lambda}(E)$ denote the E-subalgebra of $H_{\alpha}^{\Lambda}(F)$ generated by the Khovanov-Lauda generators. Then the natural map

$$
F \otimes_{E} H_{\alpha}^{\Lambda}(E) \rightarrow H_{\alpha}^{\Lambda}(F)
$$

is an F-algebra isomorphism. Moreover, letting $R_{\alpha}^{\Lambda}(E)$ be the cyclotomic Khovanov-Lauda algebra over the field $E$ defined as in \$2.2. there is an E-algebra isomorphism $R_{\alpha}^{\Lambda}(E) \stackrel{\sim}{\rightarrow} H_{\alpha}^{\Lambda}(E)$ sending the named generators of $R_{\alpha}^{\Lambda}(E)$ to the Khovanov-Lauda generators of $H_{\alpha}^{\Lambda}(E)$.

Proof. There is an obvious surjective homomorphism $R_{\alpha}^{\Lambda}(E) \rightarrow H_{\alpha}^{\Lambda}(E)$ mapping the named generators of $R_{\alpha}^{\Lambda}(E)$ to the Khovanov-Lauda generators of $H_{\alpha}^{\Lambda}(E)$. Extending scalars, this yields a map $F \otimes_{E} R_{\alpha}^{\Lambda}(E) \rightarrow H_{\alpha}^{\Lambda}(F)$. On the other hand by the presentation for $R_{\alpha}^{\Lambda}(F)$ arising from our Main Theorem, there is a map $H_{\alpha}^{\Lambda}(F) \rightarrow F \otimes_{E} R_{\alpha}^{\Lambda}(E)$ such that $e(\boldsymbol{i}) \mapsto 1 \otimes e(\boldsymbol{i})$, $y_{r} \mapsto 1 \otimes y_{r}$ and $\psi_{r} \mapsto 1 \otimes \psi_{r}$ for each $\boldsymbol{i}$ and $r$. Clearly these two maps are mutual inverses, hence both are isomorphisms. This implies that the original map $R_{\alpha}^{\Lambda}(E) \rightarrow H_{\alpha}^{\Lambda}(E)$ is injective, so it is an isomorphism, and the theorem follows.

Corollary 6.2. Let $D(F)$ be an irreducible $H_{\alpha}^{\Lambda}(F)$-module. Then there exists an irreducible $H_{\alpha}^{\Lambda}(E)$-module $D(E)$ such that $D(F) \cong F \otimes_{E} D(E)$.

Proof. By [KL1, Corollary 3.19] (extended to include the case $e=2$ as well) every irreducible $R_{\alpha}^{\Lambda}(E)$-module is absolutely irreducible.

Recall the formal character $\operatorname{ch} M$ of a finite dimensional $H_{\alpha}^{\Lambda}(F)$-module $M$ means the formal linear combination $\sum_{\boldsymbol{i} \in I^{\alpha}}\left(\operatorname{dim} M_{\boldsymbol{i}}\right) \cdot \boldsymbol{i}$, where $M_{\boldsymbol{i}}$ is the $\boldsymbol{i}$-weight space $e(\boldsymbol{i}) M \subseteq M$. It is known that two modules $M$ and $N$ are equal in the Grothendieck group if and only if $\operatorname{ch} M=\operatorname{ch} N$. Corollary 6.2 implies at once that the formal characters of the irreducible $H_{\alpha}^{\Lambda}(F)$-modules are the same as the formal characters of the irreducible $H_{\alpha}^{\Lambda}(E)$-modules (defined in an analogous way via the idempotents $\left.e(i) \in H_{\alpha}^{\Lambda}(E)\right)$. Since the formal characters of the Specht modules of [DJM] do not depend on the underlying field, this proves a conjecture of Mathas asserting that decomposition matrices of Specht modules over $H_{\alpha}^{\Lambda}(F)$ depend only on $e$ and the characteristic of $F$ (not on $F$ itself):

Corollary 6.3. Conjecture 6.38 of $[\mathrm{M}]$ holds.

Now suppose also that $K$ is a field of characteristic zero, and let $\xi \in K^{\times}$ be a primitive $e$ th root of unity if $e>0$, or some element that is not a root of unity if $e=0$. Let $H_{d}^{\Lambda}(K)$ denote the cyclotomic Hecke algebra over $K$ 
at parameter $\xi$, and $H_{\alpha}^{\Lambda}(K):=e_{\alpha} H_{d}^{\Lambda}(K)$. Fix a choice of Khovanov-Lauda generators for $H_{\alpha}^{\Lambda}(K)$ according to (4.37). The following theorem explains how $H_{\alpha}^{\Lambda}(F)$ can be obtained from $H_{\alpha}^{\Lambda}(K)$ by base change.

Theorem 6.4. Let $H_{\alpha}^{\Lambda}(\mathbb{Z})$ denote the subring of $H_{\alpha}^{\Lambda}(K)$ generated by the Khovanov-Lauda generators. Then $H_{\alpha}^{\Lambda}(\mathbb{Z})$ is a free $\mathbb{Z}$-module and there are isomorphisms

$$
\begin{aligned}
& H_{\alpha}^{\Lambda}(K) \stackrel{\sim}{\rightarrow} K \otimes_{\mathbb{Z}} H_{\alpha}^{\Lambda}(\mathbb{Z}), \\
& H_{\alpha}^{\Lambda}(F) \stackrel{\sim}{\rightarrow} F \otimes_{\mathbb{Z}} H_{\alpha}^{\Lambda}(\mathbb{Z}),
\end{aligned}
$$

such that $e(\boldsymbol{i}) \mapsto 1 \otimes e(\boldsymbol{i}), y_{r} \mapsto 1 \otimes y_{r}$ and $\psi_{r} \mapsto 1 \otimes \psi_{r}$ for each $\boldsymbol{i}$ and $r$.

Proof. $\quad$ Let $H_{\alpha}^{\Lambda}(\mathbb{Q})$ denote the $\mathbb{Q}$-subalgebra of $H_{\alpha}^{\Lambda}(K)$ generated by the Khovanov-Lauda generators. By Theorem 6.1, we can identify $H_{\alpha}^{\Lambda}(K)=$ $K \otimes_{\mathbb{Q}} H_{\alpha}^{\Lambda}(\mathbb{Q})$, and $H_{\alpha}^{\Lambda}(\mathbb{Z})$ can be viewed equivalently as the subring of $H_{\alpha}^{\Lambda}(\mathbb{Q})$ generated by its Khovanov-Lauda generators. By the same argument as in the proof of Corollary 2.2, $H_{\alpha}^{\Lambda}(\mathbb{Z})$ is spanned as a $\mathbb{Z}$-module by elements defined in terms of its Khovanov-Lauda generators like in (2.7), and only finitely many of these elements are non-zero. Hence $H_{\alpha}^{\Lambda}(\mathbb{Z})$ is a finitely generated $\mathbb{Z}$-submodule of $H_{\alpha}^{\Lambda}(\mathbb{Q})$ and it generates $H_{\alpha}^{\Lambda}(\mathbb{Q})$ over $\mathbb{Q}$. As $\mathbb{Z}$ is a principal ideal domain, this implies that $H_{\alpha}^{\Lambda}(\mathbb{Z})$ is a lattice in $H_{\alpha}^{\Lambda}(\mathbb{Q})$, hence it is also a lattice in $H_{\alpha}^{\Lambda}(K)$ i.e. it is the $\mathbb{Z}$-span of a $K$-basis of $H_{\alpha}^{\Lambda}(K)$. This shows that $H_{\alpha}^{\Lambda}(\mathbb{Z})$ is a free $\mathbb{Z}$-module and the canonical map $K \otimes_{\mathbb{Z}} H_{\alpha}^{\Lambda}(\mathbb{Z}) \rightarrow H_{\alpha}^{\Lambda}(K)$ is an isomorphism.

Now consider the $F$-algebra $F \otimes_{\mathbb{Z}} H_{\alpha}^{\Lambda}(\mathbb{Z})$. It is generated by the elements $1 \otimes e(\boldsymbol{i}), 1 \otimes y_{r}$ and $1 \otimes \psi_{r}$ for all $\boldsymbol{i}$ and $r$, and according to the Main Theorem these elements satisfy the same relations as the defining relations of the Khovanov-Lauda generators of $H_{\alpha}^{\Lambda}(F)$. Hence there is a surjection

$$
H_{\alpha}^{\Lambda}(F) \rightarrow F \otimes_{\mathbb{Z}} H_{\alpha}^{\Lambda}(\mathbb{Z}), \quad e(\boldsymbol{i}) \mapsto 1 \otimes e(\boldsymbol{i}), y_{r} \mapsto 1 \otimes y_{r}, \psi_{r} \mapsto 1 \otimes \psi_{r} .
$$

To complete the proof of the theorem, we need to show that this surjection is an isomorphism. Note that $\operatorname{dim}_{F} F \otimes_{\mathbb{Z}} H_{\alpha}^{\Lambda}(\mathbb{Z})=\operatorname{dim}_{K} H_{\alpha}^{\Lambda}(K)$ by the previous paragraph. In view of the above surjection, we know that $\operatorname{dim}_{F} H_{\alpha}^{\Lambda}(F) \geq$ $\operatorname{dim}_{K} H_{\alpha}^{\Lambda}(K)$ for all $\alpha$, and are done if we can show that equality holds everywhere. This follows because

$$
\sum_{\substack{\alpha \in Q_{+} \\ \mathrm{ht}(\alpha)=d}} \operatorname{dim}_{F} H_{\alpha}^{\Lambda}(F)=\operatorname{dim}_{F} H_{d}^{\Lambda}(F)=\operatorname{dim}_{K} H_{d}^{\Lambda}(K)=\sum_{\substack{\alpha \in Q_{+} \\ \mathrm{ht}(\alpha)=d}} \operatorname{dim}_{K} H_{\alpha}^{\Lambda}(K)
$$

by (3.6) and (4.6).

As a consequence we can explain how to reduce irreducible $H_{\alpha}^{\Lambda}(K)$-modules modulo $p$ to obtain well-defined $H_{\alpha}^{\Lambda}(F)$-modules, in the spirit of the BrauerNesbitt construction in finite group theory.

Theorem 6.5. If $D(K)$ is an irreducible $H_{\alpha}^{\Lambda}(K)$-module and $0 \neq v \in D(K)$, then $D(\mathbb{Z}):=H_{\alpha}^{\Lambda}(\mathbb{Z}) v$ is a lattice in $D(K)$ that is invariant under the action of $H_{\alpha}^{\Lambda}(\mathbb{Z})$. For any such lattice,

$$
D(F):=F \otimes_{\mathbb{Z}} D(\mathbb{Z})
$$


is naturally an $H_{\alpha}^{\Lambda}(F)$-module with the same formal character as $D(K)$. In particular, the image of $D(F)$ in the Grothendieck group is independent of the choice of lattice.

Proof. $\quad$ Let $H_{\alpha}^{\Lambda}(\mathbb{Q})$ denote the $\mathbb{Q}$-subalgebra of $H_{\alpha}^{\Lambda}(K)$ generated by the Khovanov-Lauda generators. By Corollary 6.2, there exists an irreducible $H_{\alpha}^{\Lambda}(\mathbb{Q})$-submodule $D(\mathbb{Q})$ of $D(K)$ such that $D(K)=K \otimes_{\mathbb{Q}} D(\mathbb{Q})$. We can choose this so that the given non-zero vector $v$ belongs to $D(\mathbb{Q})$. Then $H_{\alpha}^{\Lambda}(\mathbb{Z}) v$ is a finitely generated $\mathbb{Z}$-submodule of $D(\mathbb{Q})$ which must span $D(\mathbb{Q})$ over $\mathbb{Q}$ since $D(\mathbb{Q})$ is irreducible. This implies that $D(\mathbb{Z})$ is a lattice in $D(\mathbb{Q})$, hence also it is a lattice in $D(K)$. The rest of the theorem follows easily since the $\boldsymbol{i}$-weight spaces of $D(K)$ and $D(F)$ are equal to $e(\boldsymbol{i}) D(K)$ and $e(\boldsymbol{i}) D(F)$, respectively, and $e(\boldsymbol{i}) D(\mathbb{Z})$ is a lattice in $e(\boldsymbol{i}) D(K)$.

Theorem 6.5 implies that there is a well-defined notion of composition multiplicity in the reduction modulo $p$ of an irreducible $H_{d}^{\Lambda}(K)$-module. Since the irreducible $H_{d}^{\Lambda}(K)$-modules are understood by [A], it would be particularly interesting to find hypotheses on $\alpha$ that ensure that $D(F)$ is an irreducible $H_{\alpha}^{\Lambda}(F)$-module for every irreducible $H_{\alpha}^{\Lambda}(K)$-module $D(K)$. In level one, there is a precise conjecture for this known as the James conjecture; see e.g. [Ge, §2] and [F].

\section{REFERENCES}

[A] S. Ariki, On the decomposition numbers of the Hecke algebra of $G(m, 1, n), J$. Math. Kyoto Univ. 36 (1996), 789-808.

[AK] S. Ariki and K. Koike, A Hecke algebra of $(\mathbb{Z} / r \mathbb{Z})\left\{S_{n}\right.$ and construction of its irreducible representations, Advances Math. 106 (1994), 216-243.

[B] J. Brundan, Centers of degenerate cyclotomic Hecke algebras and parabolic category $\mathcal{O}$, Represent. Theory 12 (2008), 236-259.

[BK] J. Brundan and A. Kleshchev, The degenerate analogue of Ariki's categorification theorem; arXiv:0901.0057

[BKW] J. Brundan, A. Kleshchev and W. Wang, Graded Specht modules; arXiv:0901.0218

[BS] J. Brundan and C. Stroppel, Highest weight categories arising from Khovanov's diagram algebra III: category $\mathcal{O}$; arXiv:0812.1090.

[DJM] R. Dipper, G. D. James and A. Mathas, Cyclotomic q-Schur algebras, Math. Z. 229 (1998), 385-416.

[F] M. Fayers, An extension of James' conjecture, Int. Math. Res. Not. IMRN 10 (2007), 24 pp..

[Ge] M. Geck, Representations of Hecke algebras at roots of unity, Astérisque 252 (1998), 33-55.

[G] I. Grojnowski, Affine $\mathfrak{s l}_{p}$ controls the representation theory of the symmetric group and related Hecke algebras; arXiv:math.RT/9907129

[H] P. Hoefsmit, Representations of Hecke Algebras of Finite Groups with BN-Pairs of Classical Type, Ph.D. thesis, University of British Columbia, 1974.

[KL1] M. Khovanov and A. Lauda, A diagrammatic approach to categorification of quantum groups I; arXiv:0803.4121.

[KL2] M. Khovanov and A. Lauda, A diagrammatic approach to categorification of quantum groups II; arXiv:0804.2080

[K1] A. Kleshchev, Linear and Projective Representations of Symmetric Groups, Cambridge University Press, Cambridge, 2005.

[K2] A. Kleshchev, Completely splittable representations of symmetric groups, J. Algebra 181 (1996), 584-592. 
[L1] G. Lusztig, Affine Hecke algebras and their graded version, J. Amer. Math. Soc. 2 (1989), 599-635.

[L2] G. Lusztig, Introduction to Quantum Groups, Birkhäuser, 1993.

[LM] S. Lyle and A. Mathas, Blocks of cyclotomic Hecke algebras, Advances Math. 216 (2007), 854-878.

[M] A. Mathas, Iwahori-Hecke Algebras and Schur Algebras of the Symmetric Group, University Lecture Series 15, American Mathematical Society, Providence, RI, 1999.

[Ra] A. Ram, Seminormal representations of Weyl groups and Iwahori-Hecke algebras. Proc. London Math. Soc. 75 (1997), 99-133.

[Ro] J.D. Rogawski, On modules over the Hecke algebra of a p-adic group, Invent. Math. 79 (1985), 443-465.

[R1] R. Rouquier, Derived equivalences and finite dimensional algebras, Proc. ICM (Madrid 2006) 2, 191-221, EMS Publishing House, 2006.

[R2] R. Rouquier, 2-Kac-Moody algebras; arXiv:0812.5023

$[\mathrm{Ru}] \quad \mathrm{O}$. Ruff, Completely splittable representations of symmetric groups and affine Hecke algebras, J. Algebra 305 (2006), 1197-1211.

[T] W. Turner, Rock blocks, to appear in Mem. Amer. Math. Soc.; arXiv:0710.5462.

[W] H. Wenzl, Hecke algebras of Type $A_{n}$ and subfactors, Invent. Math. 92 (1988), $349-383$.

Department of Mathematics, University of Oregon, Eugene, Oregon, USA.

E-mail address: brundan@uoregon.edu, klesh@uoregon.edu 